\title{
Inertial Resonance Induced by an Oceanic Jet
}

\author{
P. KLEIN AND A. M. TREguier \\ Laboratoire de Physique des Océans, IFREMER, Plouzane, France
}

(Manuscript received 5 August 1992, in final form 19 January 1993)

\begin{abstract}
The dynamics of the mixed layer in the presence of an embedded geostrophic jet has been investigated using a simple $1 \frac{1}{2}$-layer model and a two-dimensional primitive equation model. The jet vorticity induces a spatial variability of the wind-driven inertial motions that can have some important consequences on the mixed-layer dynamics. With a steady wind stress parallel to the front, the main effect is the generation of steady upwellings and downwellings due to the divergence of the mean Ekman drift (as reported by Niiler). With a cross-front wind, however, a dramatic exponential amplification of the inertial oscillations caused by an inertial resonance mechanism is found: this mechanism can increase the inertial waves amplitude by a factor up to 10 within ten inertial periods. Competition between this resonance mechanism and the dispersion mechanisms (mainly the horizontal and vertical propagation of inertial waves) that can limit its effects has been assessed. A consequence of horizontal propagation is that energetic waves can propagate well away from the jet while continuing to absorb energy from the wind. Downward propagation disperses this energy to a depth of at least $500 \mathrm{~m}$ in a few days.
\end{abstract}

\section{Introduction}

Wind-induced inertial motions are often characterized by horizontal coherence scales much smaller than the scale of the wind. Spatial variability can be due to the wind-stress intermittence (Pollard 1990), but experimental evidence also points to a role of mesoscale oceanic currents (Weller 1982; Large et al. 1986).

The resulting spatial variability has some important consequences for mixed-layer dynamics. One consequence concerns the turbulent entrainment at the mixed-layer base that is mainly driven by the current shear instability (Price et al. 1986) and therefore by the inertial motions. This was studied by Klein and Hua (1988, 1990), who showed how mesoscale strain and vorticity can induce a spatial variability of the mixed-layer depth and of the sea surface temperature. Other consequences are linked to the resulting Ekman pumping, which produces a vertical velocity at the mixed-layer base (Niiler 1969; Rubenstein and Roberts 1986).

Another class of effects, resulting from the spatial variability of the inertial motions, concerns the vertical transfer of near-inertial energy to the thermocline and the ocean interior where it is available for mixing. This transfer depends strongly on the horizontal scales of the motion and is enhanced when small horizontal scales are present (Rubenstein 1983; Gill 1984).

Corresponding author address: Dr. Patrice Klein, Laboratoire de Physique des Océans, IFREMER, Centre de Brest, B.P. 70, 29280 Plouzane, France.
Therefore, mesoscale structures are preferential sites for the vertical energy flux because of their ability to produce small-scale inertial motions. Moreover, the energy propagation in the deep ocean can be signifcantly affected by the characteristics of the mesoscale structures as the vorticity field: this can result in a trapping and amplification of the near-inertial waves in negative vorticity regions (Kunze and Sanford 1984; Kunze 1985). Such amplification can lead to strong localized wave dissipation and mixing in the thermocline and the ocean interior.

The mechanisms that produce the shorter scales of the wind-induced inertial oscillations, or amplify them, have so far been studied using linear dynamics. In this study we have undertaken an examination of the possible role of the nonlinear terms. This has revealed some surprising results and in particular, an inertial resonance mechanism that can strongly intensify the windinduced inertial motions. The next section discusses the evolving mixed-layer spatial variability induced by the presence of a simple barotropic jet using a linear analysis. Then the role of the nonlinear terms is analyzed to show how they may trigger an inertial resonance mechanism leading, in some cases, to an exponential intensification of the inertial motions. The fourth section examines the competition between the nonlinear resonance and the dispersion processes that limit its growth, using a 11/2-layer model. In section 5 this competition and the resulting penetration of nearinertial energy within the ocean interior are studied in more detail using a two-dimensional primitive equation model. 


\section{Equations and linear analysis}

We consider the dynamics of a mixed layer embedded in a geostrophic current. To keep the problem analytically tractable, the geostrophic current is taken to be a fixed meridional jet, that is, barotropic and $y$ independent. Its velocity $V(x)$ satisfies the relation

$$
f V=\frac{\partial P}{\partial x}
$$

where $f$ is the constant Coriolis parameter and $P$ the dynamic pressure normalized by the density $\rho$. Effects of this geostrophic jet on the dynamics of the windinduced motions are first assessed using a simple $11 / 2-$ layer model. It consists of a surface mixed layer of depth $h$ capping an abyssal layer where the only motion considered is the jet velocity.

\section{a. The one-and-a-half-layer model}

The surface mixed layer is vertically homogeneous with velocities $\tilde{u}=u, \tilde{v}=v+V$ and dynamic pressure $\tilde{p}=p+P$, where $p$ is the perturbation pressure in the surface layer. The wind-stress components $\left(\tau_{x}, \tau_{y}\right)$ are normalized by $\rho$ and are constant and spatially uniform. Since the only heterogeneity comes from the geostrophic jet, $V(x)$, the $y$ derivatives are assumed negligible compared with the $x$ derivatives. Consequently, the momentum equations integrated over depth $h$ are

$$
\begin{aligned}
& h \frac{\partial \tilde{u}}{\partial t}+h \tilde{u} \frac{\partial \tilde{u}}{\partial x}-f h \tilde{v}=\tau_{x}+w_{e} \tilde{u}-h \frac{\partial \tilde{p}}{\partial x}-h D_{\tilde{u}} \\
& h \frac{\partial \tilde{v}}{\partial t}+h \tilde{u} \frac{\partial \tilde{v}}{\partial x}+f h \tilde{u}=\tau_{y}+w_{e}(\tilde{v}-V)-h D_{\tilde{v}},
\end{aligned}
$$

where $w_{e}$ is the turbulent entrainment velocity at the mixed-layer base. Since this model is not a fully stratified model, the vertical propagation of the inertial waves into the ocean interior has been taken into account through the dissipation terms $D_{\tilde{u}}$ and $D_{\tilde{v}}$. The mass conservation leads to

$$
\frac{\partial h}{\partial t}+\frac{\partial h \tilde{u}}{\partial x}=-w_{e} .
$$

The pressure gradient within the second layer is simply the geostrophic gradient $\partial P / \partial x$. So the perturbation pressure gradient in the surface layer, $\partial p / \partial x$, is given by (using the hydrostatic approximation)

$$
\frac{\partial p}{\partial x}=g \frac{\Delta \rho}{\rho} \frac{\partial h}{\partial x},
$$

with $\Delta \rho$ the density jump at the mixed-layer base. Equations (2) through (4) are simply the equations for a $11 / 2$-layer model where the mixed layer is the active layer. Note that when (2) is combined with (3), we get the following equations for the Ekman transport:

$$
\begin{gathered}
\frac{\partial h u}{\partial t}-f h v+\frac{\partial h u^{2}}{\partial x}=\tau_{x}-g \frac{\Delta \rho}{\rho} h \frac{\partial h}{\partial x}-D_{h u} \\
\frac{\partial h v}{\partial t}+\left(f+\frac{\partial V}{\partial x}\right) h u+\frac{\partial h u v}{\partial x}=\tau_{y}-D_{h v} .
\end{gathered}
$$

For the sake of simplicity, the analytical analysis performed in this section and in the next one assumes that the dispersion mechanisms as well as the entrainment at the mixed-layer base are very small. Consequently, the horizontal dispersion term (linked to $g \Delta \rho$ / $\rho)$, the dissipative terms $\left(D_{h u}\right.$ and $\left.\mathscr{D}_{h v}\right)$, and the entrainment at the ML base $\left(w_{e}\right)$ have been neglected in the next sections. Effects of these terms are considered in section 4 .

\section{b. Linear solution for the Ekman transport}

The linear solutions have already been described in the literature (for example, see Niiler 1969; Rubenstein and Roberts 1986) and are reviewed here as an introduction to the nonlinear analysis. This helps to understand how the presence of a geostrophic jet can affect the inertial motions and, in particular, induce a spatial variability of these motions. But, first, let us estimate the order of magnitude of the nonlinear terms using a dimensional analysis. We consider the initial conditions: $h=h_{i}, u=v=0$ at $t=0$. With the dispersion terms neglected, equations resulting from (5) are nondimensionalized using a length scale $L$ associated with the jet geometry, a time scale $T=1 / f$, a velocity scale

$$
U=\max \left\{\frac{\tau_{x}}{f h_{i}}, \frac{\tau_{y}}{f h_{i}}\right\},
$$

and $h_{i}$ as a depth scale. Nondimensional variables are noted by an asterisk. Let us introduce a Rossby number associated with the inertial motions defined as $\epsilon=U /$ $f L$. In this study we assume that $\epsilon \ll 1$. Note that no assumption about the jet vorticity field $\zeta=\partial V / \partial x$ (and in particular about $\zeta / f)$ is made. We use the relations $\{h u, h v\}=\left\{u^{*}, v^{*}\right\} U h_{i}$. The resulting mass equation,

$$
\frac{\partial h^{*}}{\partial t^{*}}=-\epsilon \frac{\partial u^{*}}{\partial x^{*}},
$$

shows that

$$
h^{*}=1+\epsilon h_{1}^{*}+\mathrm{O}\left(\epsilon^{2}\right) .
$$

Consequently, the equations for the Ekman transport become [using $\omega^{* 2}=1+\zeta / f$ and with the $O\left(\epsilon^{2}\right)$ terms neglected]

$$
\begin{array}{r}
\frac{\partial u^{*}}{\partial t^{*}}-v^{*}+\epsilon \frac{\partial u^{* 2}}{\partial x^{*}}=\tau_{x}^{*} \\
\frac{\partial v^{*}}{\partial t^{*}}+\omega^{* 2} u^{*}+\epsilon \frac{\partial u^{*} v^{*}}{\partial x^{*}}=\tau_{y}^{*}
\end{array}
$$


From (8), the nonlinear terms are of order $O(\epsilon)$ compared with the linear terms. Moreover, the Ekman transport equations, at the order $O(\epsilon)$, are decoupled from the $h$ equation. When only terms of order $\mathrm{O}(1)$ are considered in Eqs. (8), the resulting linear solution is (in dimensional form)

$$
\begin{aligned}
& h u=\frac{\tau_{y}}{f+\zeta}(1-\cos (\omega t))+\frac{\tau_{x}}{\omega} \sin (\omega t) \\
& h v=-\frac{\tau_{x}}{f}(1-\cos (\omega t))+\frac{\tau_{y}}{\omega} \sin (\omega t)
\end{aligned}
$$

with $\omega=[f(f+\zeta)]^{1 / 2}$.

Solution (9) shows two important effects of the jet vorticity on the Ekman transport. First, it affects the amplitude, which is larger on the negative vorticity side and smaller on the positive vorticity side. One consequence (Klein and Hua 1988) is an increase of the turbulent entrainment at the mixed-layer base on the negative vorticity side and a decrease on the positive vorticity side. Another consequence noted by Niiler (1969) is a constant upwelling or downwelling due to the divergence of the mean Ekman drift. Second, it affects the phase $(\omega t)$ of the inertial motions. Because the phase depends on $x$ [since $\omega=\omega(x)$ ], this effect amplifies with time, resulting in a linearly increasing phase difference of the inertial motions on each side of the jet. The consequence is an increasing spatial variability of the inertial motions that, as noted by Kunze (1985), can be understood using a local development of the phase $\omega t$ :

$$
\begin{aligned}
\omega(x) t & \approx \omega(0) t+\frac{\partial \omega}{\partial x}(0) x t+\cdots \\
& \approx \omega(0) t-k x
\end{aligned}
$$

where $k=-t \partial \omega / \partial x$ can be considered as a time-varying cross-front wavenumber that characterizes the spatial variability of the inertial waves. Illustration of this effect is displayed in Fig. 1, for the case of the sinusoidal front described in section 4 and for $h_{i}=30 \mathrm{~m}, \tau_{x}=0$, and $\tau_{y}=1.2 \times 10^{-4} \mathrm{~m}^{2} \mathrm{~s}^{2}$. This time-longitude plot of the $h u$ oscillations (Fig. 1a) displays a conspicuous increasing tilting of the phase lines indicating a decrease of the cross-front wavelength (Fig. 1b). The corresponding growing wavenumber $(k=-t \partial \omega / \partial x)$ has no consequence on the magnitude of the linear solutions for $h u$ and $h v$. Because of its effect on $\partial h u / \partial x$, however, it does have some consequences on the mixed-layer depth evolution.

\section{c. Linear solution for the mixed-layer depth}

From (3) (with $w_{e}=0$ ) and (9), the solution for the mixed-layer depth is

$$
\begin{aligned}
h-h_{i}=\frac{\tau_{y}}{(f+\zeta)^{2}} \frac{\partial \zeta}{\partial x} t+\frac{\partial \zeta}{\partial x} \frac{t}{2(f+\zeta)}\left(\frac{\tau_{y}}{f+\zeta} \cos (\omega t)\right. & \left.-\frac{\tau_{x}}{\omega} \sin (\omega t)\right) \\
& -\frac{\partial \zeta}{\partial x} \frac{1}{\omega(f+\zeta)}\left(\frac{3}{2} \frac{\tau_{y}}{f+\zeta} \sin (\omega t)+\frac{\tau_{x}}{\omega} \cos (\omega t)\right) .
\end{aligned}
$$
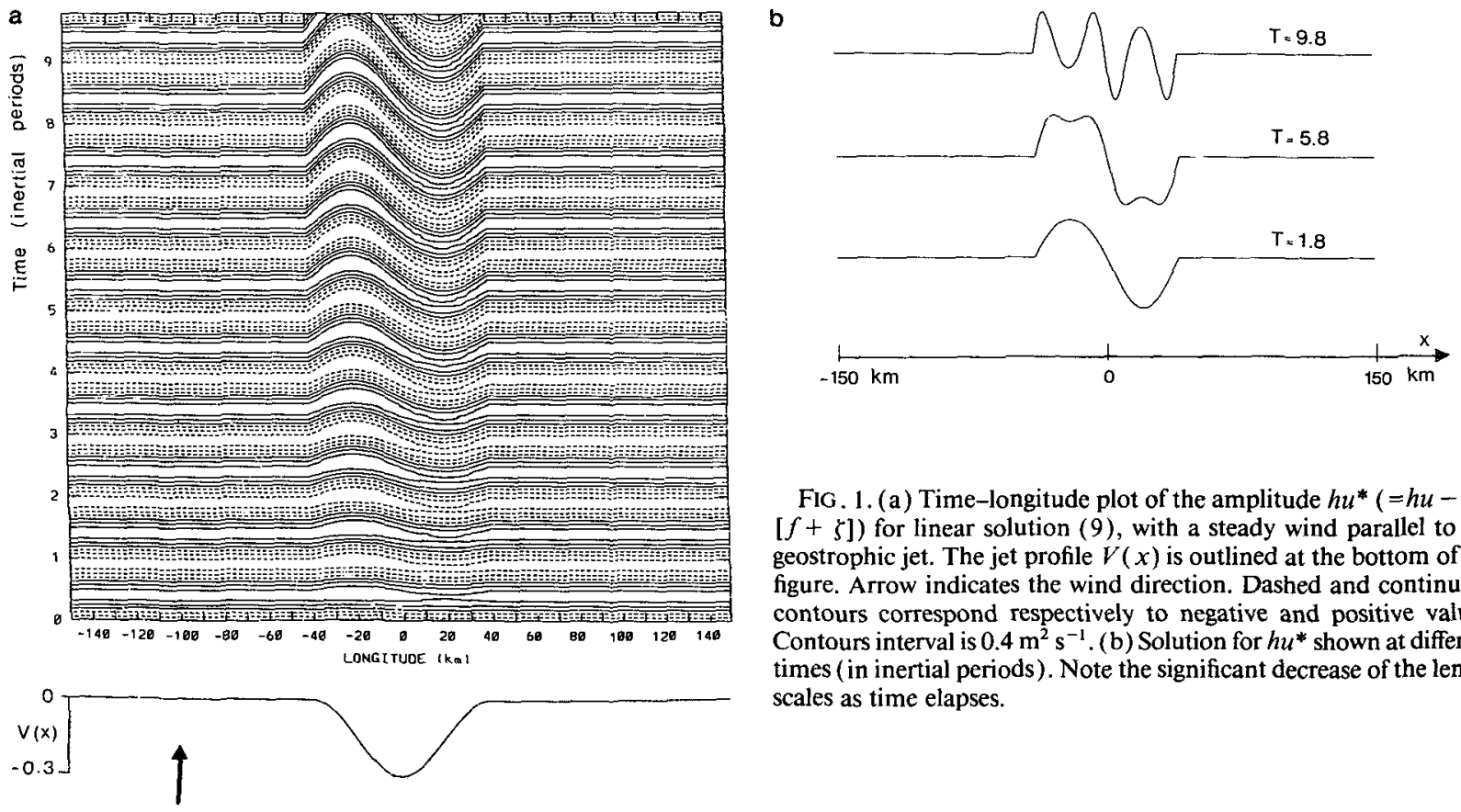

FiG. 1. (a) Time-longitude plot of the amplitude $h u^{*}\left(=h u-\tau_{y} /\right.$ $[f+\zeta]$ ) for linear solution (9), with a steady wind parallel to the geostrophic jet. The jet profile $V(x)$ is outlined at the bottom of the figure. Arrow indicates the wind direction. Dashed and continuous contours correspond respectively to negative and positive values. Contours interval is $0.4 \mathrm{~m}^{2} \mathrm{~s}^{-1}$. (b) Solution for $h u^{*}$ shown at different times (in inertial periods). Note the significant decrease of the length scales as time elapses. 

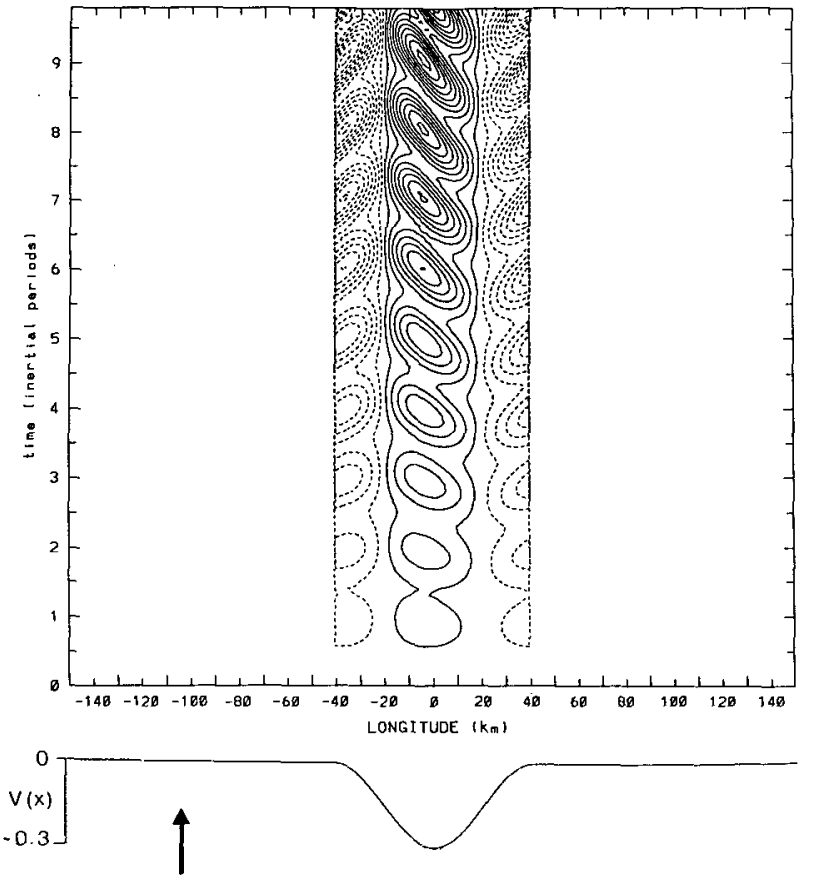

FIG. 2. Time-longitude plot of the mixed-layer depth perturbation ( $h^{*}=h-h_{i}$ with $h_{i}=30 \mathrm{~m}$ ) for linear solution (11), with a steady wind parallel to the jet. Dashed and continuous contours correspond, respectively, to negative and positive values. Contours interval is 1 m.

This solution is characterized by two dominant features that grow linearly with time. The first one comes from the first right-hand side time-dependent term, that is,

$$
\frac{\tau_{y}}{(f+\zeta)^{2}} \frac{\partial \zeta}{\partial x} t \text {. }
$$

It is a systematic upwelling or downwelling that works only when the wind has a nonzero component parallel to the front $\left(\tau_{y} \neq 0\right)$, and depends on the sign of $\tau_{y} \partial \zeta /$ $\partial x$ : for a southward jet and northward wind (Fig. 2) there is downwelling in the middle of the jet and upwelling on the edges. Using the values $f=10^{-4} \mathrm{~s}^{-1}$, $\tau_{y}=1.2 \times 10^{-4} \mathrm{~m}^{2} \mathrm{~s}^{-2}, \partial \zeta / \partial x \approx 10^{-9} \mathrm{~m}^{-1} \mathrm{~s}^{-1}$, the estimated mixed-layer depth variation is $7.5 \mathrm{~m}$ after 10 inertial periods (Fig. 2). This corresponds to a vertical velocity of $1 \mathrm{~m}^{\text {day }}{ }^{-1}$. The spatial variability associated with this feature is related to the jet vorticity gradient (Niiler 1969). The second feature, related to the next two right-hand side terms of (11), comes from the jet vorticity effect on the phase of the inertial motions and is nonzero whatever the wind direction is. It appears in Fig. 2 as an inertial oscillation of the mixedlayer depth, that is, a sequence of upwellings and downwellings that linearly increase with time.

Because of the growing spatial variability of the linear solution, nonlinear terms in (8) involving horizontal gradients cannot be neglected for times large compared with the inertial period. We first investigated these nonlinear effects numerically. For a wind parallel to the front (i.e., $\tau_{x}=0$ and $\tau_{y} \neq 0$ ), the solution was qualitatively similar to the linear one. With a crossfront wind (i.e., $\tau_{x} \neq 0$ and $\tau_{y}=0$ ), however, we found a dramatic increase of the amplitude of the inertial oscillations. The resulting mixed-layer depth variation reaches $31 \mathrm{~m}$ after 10 inertial periods (Fig. 3) for the same parameters (except for the wind direction) as the case displayed in Fig. 2. We have found that this exponential growth is due to a nonlinear resonance. A first insight into this mechanism can be obtained through a perturbation analysis.

\section{Perturbation analysis of the nonlinear equations}

\section{a. $O(\epsilon)$ solution for the Ekman transport}

Equations ( 8 ) for $u^{*}$ and $v^{*}$, the nondimensionalized Ekman transport components, are considered with the $O(\epsilon)$ nonlinear terms included. The following analysis assumes that $\epsilon$ is small (i.e., $\epsilon=U / f L \ll 1$ ) and moreover that $\epsilon$ is small compared with $\zeta / f$. We look for an estimation of the solution of (8) at times larger than $f^{-1}$. Henceforth, asterisks are dropped for nondimensional variables. Dimensional expressions will be explicitly mentioned when they are introduced.

Equations ( 8 ) describe a nonlinear oscillator with natural frequency $\omega$. Therefore, as a basic assumption, we consider the components of the nonlinear terms with frequency $\omega$ as the most important ones for the long-term behavior of the solution since they can induce resonance. Let us define the new variables $u^{\prime}$ and $v^{\prime}$ as

$$
u=\frac{\tau_{y}}{\omega^{2}}+u^{\prime}, v=-\tau_{x}+v^{\prime}
$$

Then, using these new variables, the nonlinear terms can be linearized in the following way:

$$
\begin{gathered}
\frac{\partial u^{2}}{\partial x} \approx 2 \frac{\tau_{y}}{\omega^{2}} \frac{\partial u^{\prime}}{\partial x}+2 u^{\prime} \frac{\partial \frac{\tau_{y}}{\partial x}}{\omega^{2}} \\
\frac{\partial u v}{\partial x} \approx \frac{\tau_{y}}{\omega^{2}} \frac{\partial v^{\prime}}{\partial x}+v^{\prime} \frac{\partial \frac{\tau_{y}}{\omega^{2}}}{\partial x}-\tau_{x} \frac{\partial u^{\prime}}{\partial x} .
\end{gathered}
$$

In other words, the decomposition (12) assumes that a single wave dominates, so that the wave-wave interaction terms drop out. Using (12), the nonlinear equations (8) are reduced to the simplified linearized equations:

$$
\begin{array}{r}
\frac{\partial u^{\prime}}{\partial t}-v^{\prime}+2 \epsilon \frac{\tau_{y}}{\omega^{2}} \frac{\partial u^{\prime}}{\partial x}+2 \epsilon u^{\prime} \frac{\partial \frac{\tau_{y}}{\partial x}}{\partial x}=0 \\
\frac{\partial v^{\prime}}{\partial t}+\omega^{2} u^{\prime}+\epsilon \frac{\tau_{y}}{\omega^{2}} \frac{\partial v^{\prime}}{\partial x}+\epsilon v^{\prime} \frac{\partial \frac{\tau_{y}}{\partial x}}{\partial x}-\epsilon \tau_{x} \frac{\partial u^{\prime}}{\partial x}=0,
\end{array}
$$



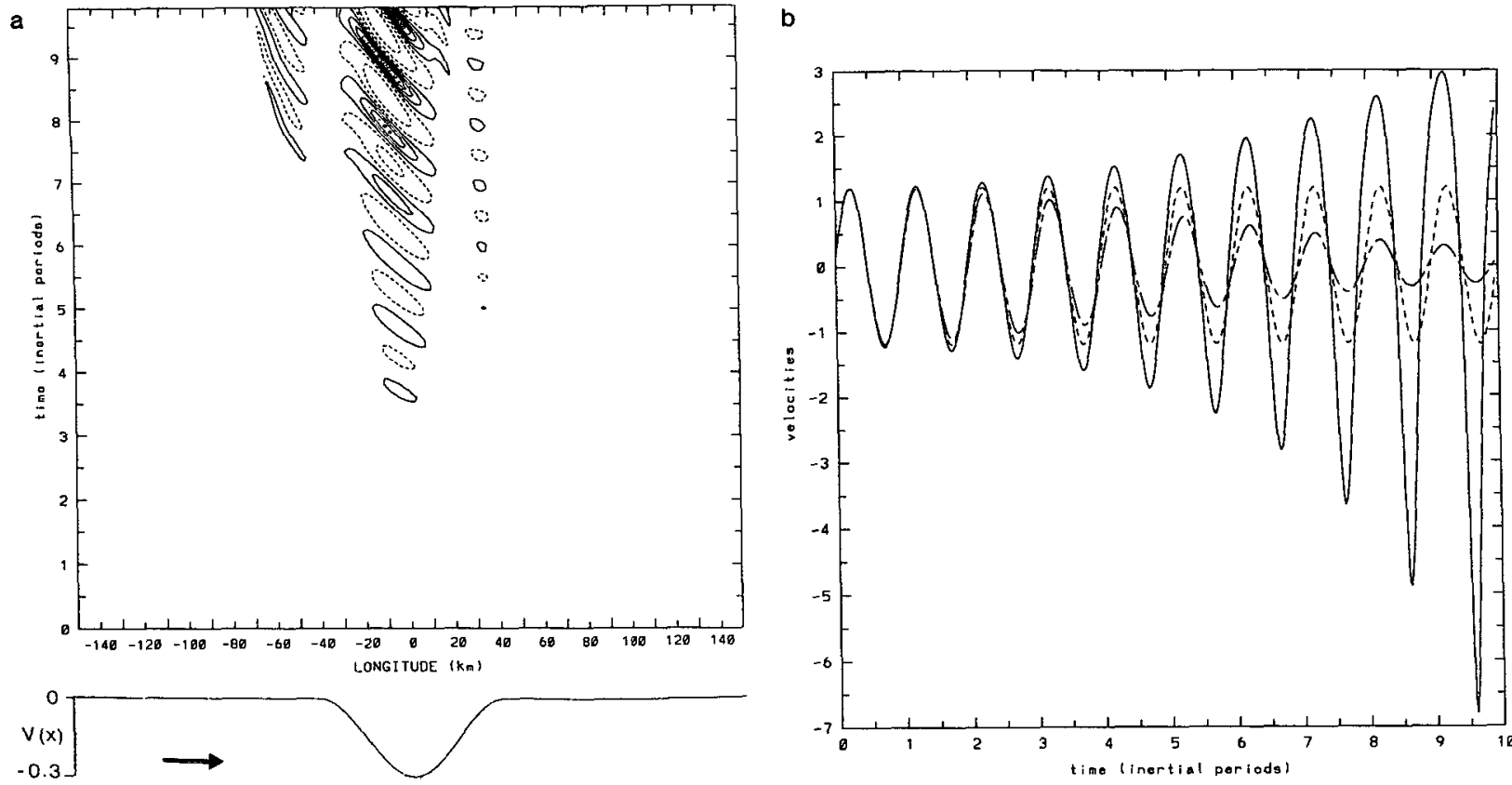

FIG. 3. (a) Time-longitude plot of the mixed-layer depth perturbation ( $h^{*}=h-h_{i}$ with $h_{i}=30 \mathrm{~m}$ ) for a nonlinear solution with steady wind perpendicular to the jet. Contours interval is $3 \mathrm{~m}$. (b) Time series of $h u$ at three longitudes for the nonlinear solution with a wind perpendicular to the jet: $x=-110 \mathrm{~km}$ (dashed), $x=-30 \mathrm{~km}$ (dot dashed), $x=0 \mathrm{~km}$ (solid).

which can be rewritten as

$\frac{\partial^{2} u^{\prime}}{\partial t^{2}}+\omega^{2} u^{\prime}+\epsilon\left(3 \frac{\tau_{y}}{\omega^{2}} \frac{\partial^{2} u^{\prime}}{\partial t \partial x}+3 \frac{\partial \frac{\tau_{y}}{\omega^{2}}}{\partial x} \frac{\partial u^{\prime}}{\partial t}-\tau_{x} \frac{\partial u^{\prime}}{\partial x}\right)=0$

Now let us search for solutions like

$$
\left[u^{\prime}, v^{\prime}\right]=\left[u_{0}, v_{0}\right] e^{-i\left[\omega t+\epsilon f_{1}+O\left(\epsilon^{2}\right)\right]},
$$

where $f_{1}(x, t)$ is a phase perturbation introduced to take into account the effects of the $O(\epsilon)$ terms. A solution for $f_{1}$ that satisfies (14) is given in appendix $\mathrm{A}$ for a general case that includes dispersion. In the present case, in which the dispersion mechanisms are neglected, use of (15) into (14) leads to

$$
\epsilon f_{1}=-\epsilon \frac{3}{4} \frac{\tau_{y}}{\omega^{2}} \frac{\partial \omega}{\partial x} t^{2}+i \epsilon\left(3 \frac{\tau_{y}}{\omega^{3}} \frac{\partial \omega}{\partial x} t+\frac{\tau_{x}}{4 \omega} \frac{\partial \omega}{\partial x} t^{2}\right) .
$$

This solution displays some very important effects linked to the nonlinear terms of the Ekman transport equations. First, it should be noted that the three terms on the right-hand side of (16) involve the jet vorticity gradient (included in $\partial \omega / \partial x$ ). More precisely, they all involve the across-front wavenumber $k=-t \partial \omega / \partial x$ and therefore are clearly linked to the spatial variability of the Ekman transport components. The first right-handside term is a modification of the frequency "seen" by the inertial motions that can be simply interpreted as a Doppler shift due to the zonal mean Ekman drift $\tau_{y} /$ $\omega^{2}$; it can be rewritten as

$$
\frac{3}{4} \epsilon \frac{\tau_{y}}{\omega^{2}} k,
$$

with $k$ the across-front wavenumber. Using the values of section 2, this Doppler shift, which in dimensional form is

$$
-\frac{3}{8} \frac{\tau_{y}}{h_{i}(f+\zeta)} \frac{\partial \zeta}{\partial x} t
$$

attains $\approx 0.1 f$ after 8 days and does not affect much the frequency. The second term corresponds to an exponential growth or decay due to the divergence of the zonal-mean Ekman drift. The corresponding growth or decay rate is constant and in dimensional units is

$$
\approx \frac{3}{2 h_{i}} \frac{\tau_{y}}{(f+\zeta)^{2}} \frac{\partial \zeta}{\partial x} \text {. }
$$

It is usually small $\left(\approx 10^{-6} \mathrm{~s}^{-1}\right)$. The third term produces a much more significant growth or decay of the Ekman transport since the corresponding growth rate grows linearly in time. Therefore, the solution behaves like $\exp \left(t^{2}\right)$. In dimensional units, the corresponding growth rate is

$$
\frac{\tau_{x}}{8 h_{i}(f+\zeta)} \frac{\partial \zeta}{\partial x} t
$$

Using the values of section 2 , this leads to the tenfold increase of the amplitude of the oscillating part of the Ekman transport after 8 days seen in Fig. 3.

The term involving $\tau_{x}$ comes from the linearization of the nonlinear term $\partial u v / \partial x$ [see (12)] and acts in 
(14) as a forcing term that induces resonance. Hence, the corresponding mechanism is called an inertial resonance mechanism.

\section{b. Discussion on the inertial resonance mechanism}

The inertial resonance mechanism can be interpreted physically in a simple way. From the findings of section 2 , the main role of the geostrophic jet is to produce a mesoscale variability of the inertial motions. So let us look at a situation without a geostrophic jet, but where the inertial oscillations vary in space with a constant zonal wavenumber $k$. For clarity, we consider only the case $\tau_{y}=0$, without entrainment, dissipation, or propagation.

Let us show first how an equation very similar to (14) can be derived directly from the momentum equations (instead of the transport equations). First, it is important to note that, from the basic assumption used in section 3a [leading to (12)], the nonlinear advection terms in Eqs. (2) and (3) cannot induce resonance and therefore can be neglected. The resulting simplified equations become (in dimensional form)

$$
\begin{array}{r}
\frac{\partial u}{\partial t}-f v=\frac{\tau_{x}}{h} \\
\frac{\partial v}{\partial t}+f u=0 \\
\frac{\partial h}{\partial t}+h_{i} \frac{\partial u}{\partial x}=0 .
\end{array}
$$

If we take the derivative of $(17)$ with respect to time we obtain [using (18)]

$$
\frac{\partial^{2} u}{\partial t^{2}}+f^{2} u-h_{i} \frac{\tau_{x}}{h^{2}} \frac{\partial u}{\partial x}=0
$$

From $h_{i}$, the initial mixed-layer depth, and $L$, the length scale associated with $k$, a dimensional analysis that uses

$$
\epsilon=\frac{\tau_{x}}{f^{2} h_{i} L} \ll 1
$$

reveals that the term involving $\tau_{x}$ in (19) is of order $\mathrm{O}(\epsilon)$ compared with the others and that the $h$ variations are of order $\mathrm{O}(\epsilon)$ compared with $h_{i}$. Consequently the last term in (19) can be approximated as

$$
h_{i} \frac{\tau_{x}}{h^{2}} \frac{\partial u}{\partial x} \approx \frac{\tau_{x}}{h_{i}} \frac{\partial u}{\partial x}
$$

A simple linear equation results:

$$
\frac{\partial^{2} u}{\partial t^{2}}+f^{2} u-\frac{\tau_{x}}{h_{i}} \frac{\partial u}{\partial x}=0
$$

This equation resembles the nondimensional equation (14) with $\tau_{y}=0$. Deriving a dispersion relation for solutions proportional to $\exp [i(k x-\omega t)]$, we find a similar instability growth rate, that is, $k \tau_{x} /\left(f h_{i}\right)$. Thus, the solutions with $k \tau_{x}<0$ grow exponentially while those with $k \tau_{x}>0$ are damped. This means that waves propagating against the wind amplify as they propagate. Waves propagating in the same direction as the wind decay.

The derivation of the dimensional equation (20) does not yield new results but it allows a much better understanding of the instability. Indeed, the term responsible for growth $\left[\left(\tau_{x} / h_{i}\right) / \partial u / \partial x\right]$ simply comes from the term $\tau_{x} / h$ present in the momentum equations (17). So let us examine the role of the $\tau_{x} / h$ term in these equations. We use $\tau_{x}>0$. First, consider the case of linear inertial oscillations involving the forcing term $\tau_{x} / h_{i}$ instead of $\tau_{x} / h$ (Fig. 4a). The velocity $u$ is alternately positive and negative with the same magnitude. Therefore, the energy flux from the wind, $u \tau_{x}$, changes sign during an inertial period. The time average is exactly zero and there is no net energy gain. When the forcing is $\tau_{x} / h$ instead of $\tau_{x} / h_{i}$, the phase relationship of $u$ and $h$ must be taken into account. For negative wavenumbers $(k<0), h$ is minimum when $u$ is positive and maximum, as shown in Fig. $4 \mathrm{~b}$. So the forcing $\tau_{x} / h$ represented by a big arrow is largest when it is in the direction of $u$ and provides more energy to the system than when $h=h_{i}$. On the other hand, $h$ is maximum when $u$ is in the opposite direction from the wind. So, at that time, the forcing $\tau_{x} / h$ is smaller than when $h=h_{i}$, and consequently the wind removes

(a) linear case
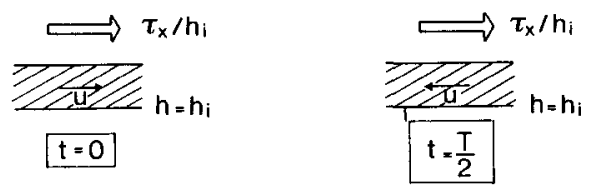

(b) nonlinear $k \tau_{x}<0$
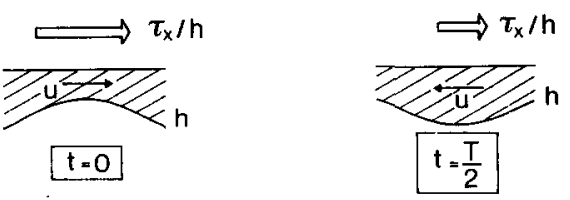

(C) nonlinear $k \tau_{x}>0$
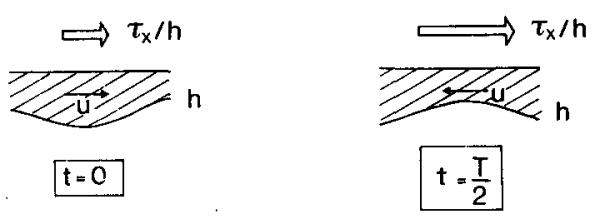

FIG. 4. Sketch of the inertial resonance mechanism. Here $T$ represents an inertial period. 
less energy. On average over an inertial period, more energy is input than removed and there is a net growth of the oscillation when $k \tau_{x}<0$. For a positive wavenumber $k$, the phase relationship of $u$ and $h$ is reversed (Fig. 4c): $h$ is larger when $u$ is in the direction of the wind, decreasing the input of energy, and $h$ is smaller when $u$ opposes the wind stress, increasing the removal of energy. Hence, the decay of the oscillations when $k \tau_{x}>0$.

The resonance phenomenon may be observed for small-scale waves when the wind is large and the mixedlayer depth is small. For example, with $k=10^{-4} \mathrm{~m}^{-1}$, $\tau_{x}=10^{-4} \mathrm{~m}^{2} \mathrm{~s}^{-2}$, and $h_{i}=30 \mathrm{~m}$, the growth rate $\left[k \tau_{x}\right]$ $\left.\left(f h_{i}\right)\right]$ is about $3.5 \mathrm{day}^{-1}$. The presence of a geostrophic jet is not necessary for inertial resonance to happen. It renders the phenomenon more dramatic, however, since the initial growth is faster than $e^{t}$ due to the wavenumber of the inertial oscillations that grows with time.

\section{Numerical solution of the nonlinear equations}

Both the analytical solution (16) and the solution of appendix A are crude approximations of the full solution, which has to be found by direct numerical integrations of the full equations ( 3 ) and (5). These numerical integrations have been performed using the following geostrophic jet:

$$
V(x)=\left\{\begin{array}{cc}
-V_{0}\left[1+\cos \left(\frac{2 \pi}{\lambda} x\right)\right], & |x|<\lambda / 2 \\
0, & |x| \geqslant \lambda / 2,
\end{array}\right.
$$

with $V_{0}=0.15 \mathrm{~m} \mathrm{~s}^{-1}$ and $\lambda=80 \mathrm{~km}$. This jet is the same as the one considered by Rubenstein and Roberts (1986). Its maximum velocity is $0.30 \mathrm{~m} \mathrm{~s}^{-1}$. Other simulations performed with an exponential jet are very similar with the ones examined in this paper, and hence it appears that qualitative results are not sensitive to the jet geometry. The following values for other parameters have been used: $f=10^{-4} \mathrm{~s}^{-1}, h_{i}=30 \mathrm{~m}, \tau_{x}$, $\tau_{y}=0$ or $1.2 \times 10^{-4} \mathrm{~m}^{2} \mathrm{~s}^{-2}, u_{i}=0$, and $v_{i}=0$. Equations ( 3 ) and (5) have been discretized using standard numerical methods: a leapfrog scheme in time and centered finite differences in the $x$ direction. Note that a high resolution is necessary since the solution evolves toward smaller and smaller scales. The domain is periodic, of length $300 \mathrm{~km}$, spatial resolution is $\Delta x$ $=500 \mathrm{~m}$ and the time step is $\Delta t=65 \mathrm{~s}$.

The horizontal propagation resulting from horizontal pressure gradients is treated explicitly in the $11 / 2-$ layer model. It depends on the parameter $g \Delta \rho / \rho$. Nearinertial waves with zonal wavenumber $k$ horizontally propagate with a group velocity $C_{g x}$ whose magnitude is

$$
\approx g \frac{\Delta \rho}{\rho} h \frac{k}{f},
$$

and with a direction that depends on the sign of $k$. Vertical propagation on the other hand is impossible, because no perturbation is allowed below the active layer. This effect must therefore be parameterized (through $\mathscr{D}_{h u}$ and $\mathscr{D}_{h v}$ ). Our choice of a Laplacian friction is explained in appendix B. The amount of energy dissipated by the Laplacian friction (calculated as shown in the appendix) is used as an estimate of the amount of energy that would be radiated downward in a fully stratified model. Finally, a simple representation of entrainment (chosen as in Klein and Hua 1988 ) has been added. Its parameterization involves a critical mixed-layer depth $h_{c o}$ ( see appendix B). In order to isolate the effects of the nonlinear terms and in particular the inertial resonance, some simulations have been performed with the entrainment velocity $\left(w_{e}\right)$ set equal to zero and with nonzero but very small values for the density jump at the mixed-layer base and for the friction coefficient. These very small values are $g \Delta \rho / \rho=2 \times 10^{-4} \mathrm{~m} \mathrm{~s}^{-2}$ and $\nu=10 \mathrm{~m}^{2} \mathrm{~s}^{-1}$. Other simulations have been performed with more realistic values for $\Delta \rho$ and $\nu$ and a nonzero $w_{e}$ in order to investigate the competition between inertial resonance and dispersion mechanisms. These "realistic values" are $\nu=40 \mathrm{~m}^{2} \mathrm{~s}^{-1}, g \Delta \rho / \rho=1.5 \times 10^{-3} \mathrm{~m} \mathrm{~s}^{-2}$, and we use $h_{c o}=20 \mathrm{~m}$ when $w_{e} \neq 0$. The two situations examined are a wind stress parallel to the jet $\left(\tau_{x}=0\right.$ and $\tau_{y}=1.2 \times 10^{-4} \mathrm{~m}^{2} \mathrm{~s}^{-2}$ ) and a wind stress perpendicular to the jet $\left(\tau_{x}=1.2 \times 10^{-4} \mathrm{~m}^{2} \mathrm{~s}^{-2}\right.$ and $\left.\tau_{y}=0\right)$.

\section{a. Wind stress parallel to the jet}

In this case, with very small values for $g \Delta \rho / \rho$ and $\nu$ and $w_{e}=0$, the nonlinear solution (not shown) resembles the linear one (Figs. 1 and 2), displaying in particular a systematic downwelling at the center of the jet and upwellings on the edges for the mixed-layer depth.

With more realistic values for $g \Delta \rho / \rho$ and $\nu$ and a nonzero entrainment velocity, numerical results again do not show significant differences with the linear solution. The only differences, displayed by the comparison of Fig. 5 with Fig. 1, concern a small local intensification of $h u$ values at the jet center and a conspicuous depletion on the right side of the jet. These differences can be explained in terms of the competition between the mean Ekman drift and the group velocity associated to the horizontal wave propagation $\left(C_{g x}\right)$. Because of the $k$ expression

$$
\left(k \approx-\frac{1}{2} \frac{\partial \zeta}{\partial x} t\right), C_{g x}\left(\approx g \frac{\Delta \rho}{\rho} h \frac{k}{f}\right)
$$

increases with time and moreover is directed westward at the jet center and eastward on the edges. Consequently, at the jet center, the horizontal propagation counters and, later on, overcomes the eastward mean Ekman drift. As a result, waves there are first locally stationary and later are expelled to the left side of the 

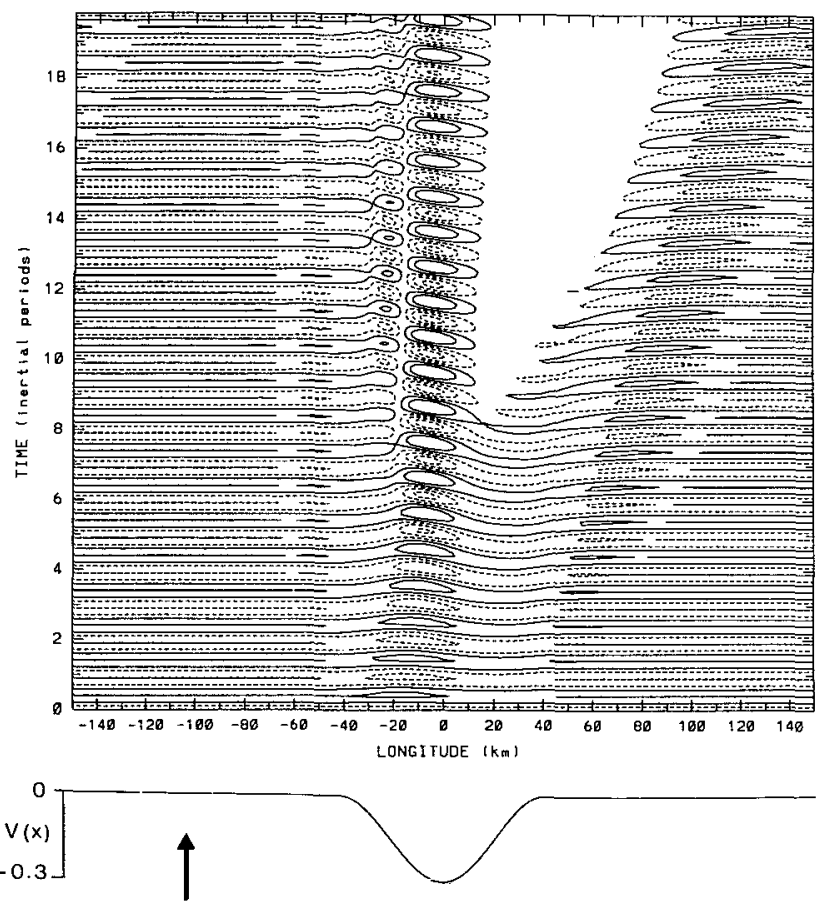

FIG. 5. Time-longitude plot of the amplitude $h u^{*}\left(=h u-\tau_{y} /[f\right.$ $+\zeta])$ for the nonlinear solution with a wind parallel to the geostrophic jet, and with the dispersion mechanisms taken into account. Dashed and continuous contours correspond, respectively, to negative and positive values. Contours interval is $0.8 \mathrm{~m}^{2} \mathrm{~s}^{-1}$.

jet where they seem to be trapped. On the right edge the eastward advection by the mean Ekman drift dominates. So waves are quickly expelled, and since there is no energy flux from the center this place becomes energy depleted. Then, with time, the energy depleted area extends far eastward out of the jet. Time evolution of the kinetic energy integrated over the whole domain does not show significant evolution (Fig. 8). The only change is a slight decrease of the kinetic energy in the mixed layer, due to the vertical propagation of kinetic energy in the bottom layer.

\section{b. Wind stress perpendicular to the jet}

In this case, with very small values for $g \Delta \rho / \rho$ and $\nu$ and $w_{e}=0$, the nonlinear numerical solution (Fig. 3a) differs dramatically from the linear one. The nonlinear case displays a strong increase of the amplitude of the oscillating Ekman transport. This increase is located at the center of the jet (Fig. 3b) where the wavenumber $k\left(\approx-\frac{1}{2} \partial \zeta / \partial x\right)$, that is, where $\tau_{x} k<0$ (since $\partial \zeta / \partial x$ is positive in the middle of the jet). This leads to a phase velocity opposite to the wind-stress direction. At this location, the extreme $h u$ values have an exponential-like behavior (Fig. 3b) with a much larger increase for the negative values (more than a ten times increase after 10 inertial periods). This asymmetry is reflected in the $h$ evolution (Fig. 3a), which exhibits much more intense downwelling than upwelling. This asymmetry was not predicted by the analytical solution. On the edges of the jet, where $k$ is positive ( since $\partial \zeta / \partial x<0$ ), the oscillating components of the Ekman transport are strongly damped (Fig. 3b). The opposite situation (not shown), that is, a growth on the edges and a damping in the middle of the jet, is found when the wind direction is reversed. This striking behavior is best illustrated by growth of the energy integrated over the whole domain: we have found that its value at 10 inertial periods is more than two times larger than its value at 2 inertial periods, demonstrating the inertial resonance mechanism.

With more realistic values for $g \Delta \rho / \rho$ and $\nu$ and with $w_{e} \neq 0$, the inertial resonance mechanism is still very efficient despite the strong influence of the dispersion mechanisms on the oceanic response. A particularly remarkable new feature of this response is the westward propagation of the inertial waves, which keep growing far out of the jet (Fig. 6). The growth of these inertial waves outside the jet, however, is not as large as the one found previously: at 10 inertial periods, maximum $h u$ value is $2.5 \mathrm{~m}^{2} \mathrm{~s}^{-1}$ instead of $6 \mathrm{~m}^{2} \mathrm{~s}^{-1}$ found in section 4. Let us explain these features in terms of the competition between the resonance mechanism and the dispersion.

From section $3 b$, the exponentially growing waves are the ones with a wavenumber $k$ such that $\tau_{x} k<0$, that is, $k<0$ in this wind-stress situation. Therefore,

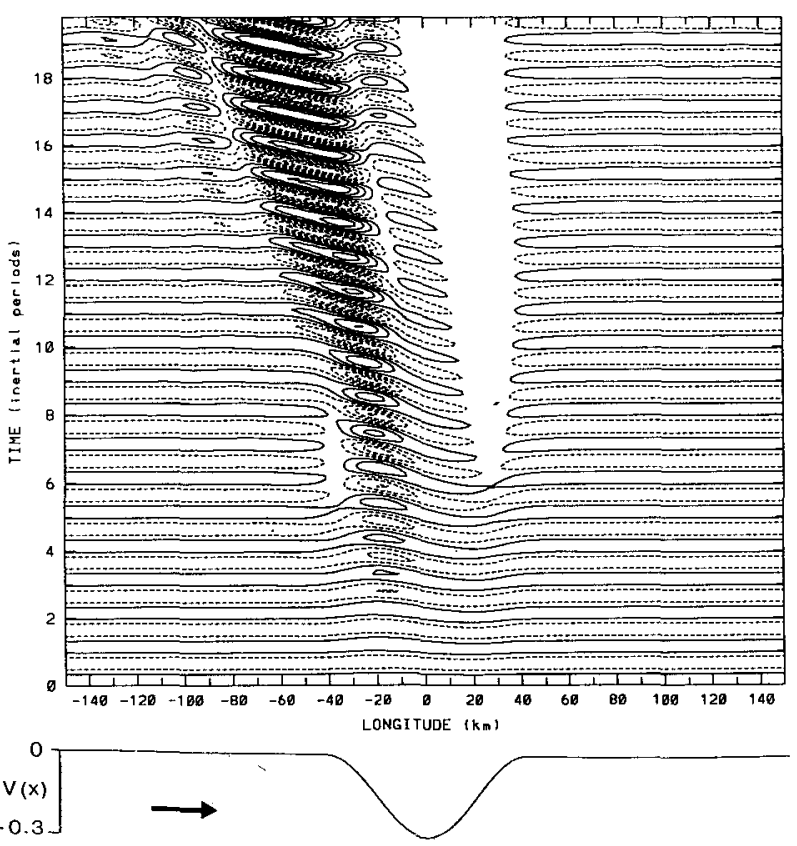

FIG.6. Time-longitude plot of the amplitude $h u$ for the nonlinear solution with a wind perpendicular to the jet, and with the dispersion mechanisms taken into account. Dashed and continuous contours correspond respectively to negative and positive values. Contours interval is $1 \mathrm{~m}^{2} \mathrm{~s}^{-1}$. 
these energetic waves should originate at the jet center (where $k<0$ ) and then propagate westward, while on the edges of the jet (where $k>0$ ) waves are quickly damped. This is what happens from Fig. 6, which reveals that the energetic waves originated at the jet center are strong enough to go across the left edge and to keep propagating westward outside of the jet. So, at the jet center, the exponential growth effect is limited by the horizontal propagation that disperses the waves. The time when the horizontal propagation balances the resonant growth at the jet center can be estimated using the damping rate due to horizontal wave propagation:

$$
r_{h}=k C_{g x} \approx g \frac{h}{2} \frac{\Delta \rho}{\rho} \frac{k^{2}}{f} .
$$

The balance occurs for a critical wavenumber

$$
k_{c} \approx \frac{6}{8} \frac{\tau}{f h^{2}} / \frac{g \Delta \rho}{\rho} .
$$

From the $k$ expression $\left(k \approx-\frac{1}{2}(\partial \zeta / \partial x) t\right)$, this balance should happen when $t>5.1$ inertial periods. This value agrees well with the one from numerical results $(\approx 5.4)$. On the other hand, from the theoretical solution of section $3 \mathrm{~b}$, westward-propagating waves (that correspond to $\tau_{x} k<0$ ) should keep growing outside the jet. This is actually what Fig. 6 shows. In this region, however, their growth is limited by the turbulent entrainment at the mixed-layer base and the Laplacian friction.
Since these two dispersion mechanisms are not too large, the waves keep growing.

Time evolution of the kinetic energy of the oscillating part of the inertial motions (Fig. 7a), integrated within the mixed layer and averaged over one inertial period, well confirms the preceding features. A strong kinetic energy increase (from $2.4 \times 10^{-2} \mathrm{~m}^{3} \mathrm{~s}^{-2}$ up to a maximum of $4.0 \times 10^{-1} \mathrm{~m}^{3} \mathrm{~s}^{-2}$ within 20 inertial periods) appears on the negative vorticity side and extends outside the jet (on the left side), while a relative depletion appears in the positive vorticity region. The kinetic energy dissipated by the Laplacian friction (assumed to represent the downward injection into the deeper layer) has been calculated (see appendix B) and is shown in Fig. 7b: the downward kinetic energy injection occurs in the negative vorticity region and extends on the left side of the jet, that is, where the surface inertial energy is maximum. The maximum value attains $1.45 \times 10^{-1} \mathrm{~m}^{3} \mathrm{~s}^{-2}$, that is, almost five times the value in the upper layers when no jet is present. Time evolution of the total kinetic energy integrated over the whole domain (Fig. 8) confirms the importance of the resonance mechanism even in the presence of dispersion: after 20 inertial periods, the total kinetic energy in the surface layer attains $6.7 \times 10^{-2} \mathrm{~m}^{3} \mathrm{~s}^{-2}$, almost four times the corresponding energy when the wind stress is parallel to the jet! Furthermore, the kinetic energy injected in the deeper layer (estimated through the Laplacian friction) is more than 1.5 times the total a
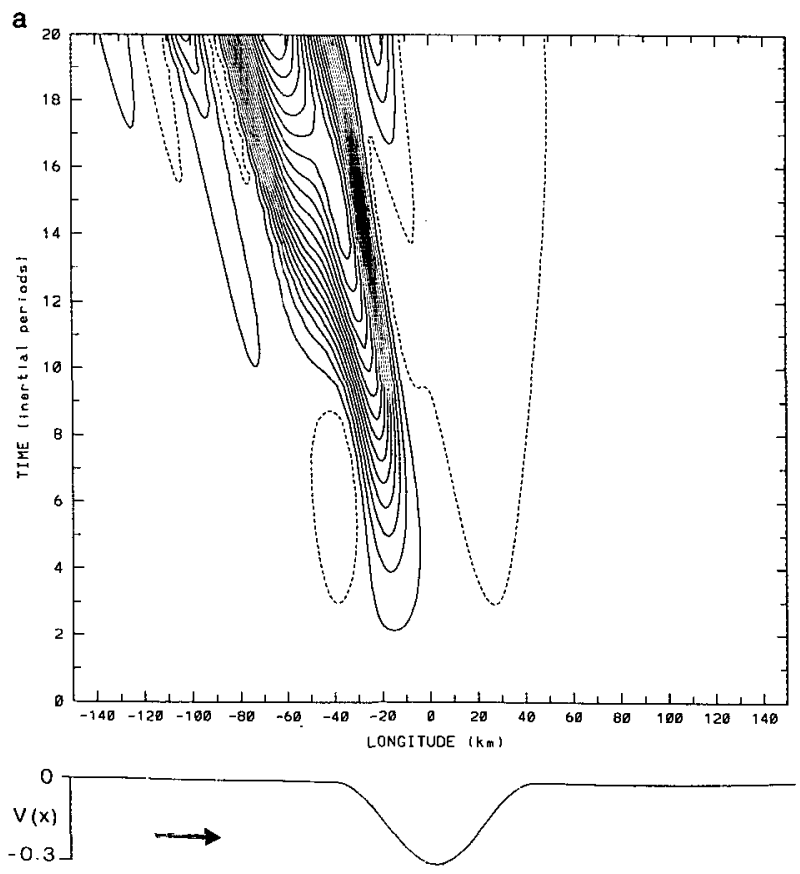

b

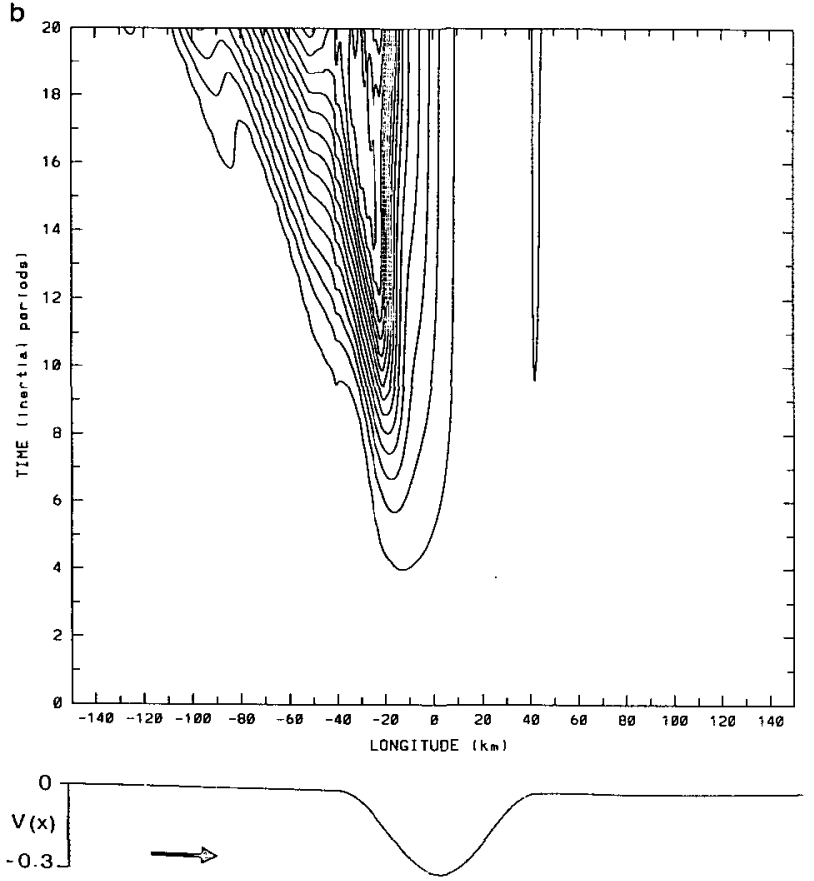

FIG. 7. Time-longitude plot (a) of the kinetic energy (oscillating part of the inertial motions) perturbation relatively to a mean value $\left(h\left(u^{2}+v^{2}\right) / 2-\left\langle h\left(u^{2}+v^{2}\right)\right\rangle / 2\right)$, and (b) of the kinetic energy injected in the bottom layer for a wind stress perpendicular to the jet. Dashed and continuous contours correspond, respectively, to negative and positive values. Contours intervals are $0.02 \mathrm{~m}^{3} \mathrm{~s}^{-2}$ in (a) and $0.01 \mathrm{~m}^{3} \mathrm{~s}^{-2}$ in (b). Mean value in (a), equal to $2.4 \times 10^{-2} \mathrm{~m}^{3} \mathrm{~s}^{-2}$, corresponds to the value when no jet is present. 


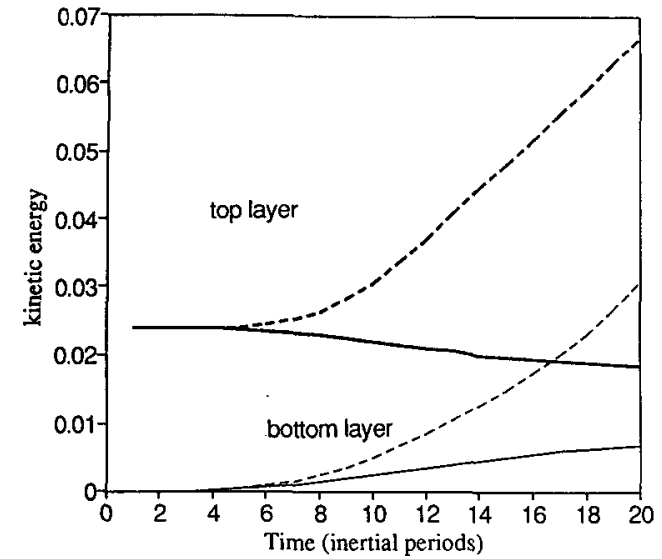

FIG. 8. Time series of kinetic energy (oscillating part of the inertial motions) obtained from the nonlinear solution with the dispersion mechanisms taken into account. Thick lines correspond to the kinetic energy integrated within the mixed layer and averaged in the $x$ direction and thin lines to the kinetic energy integrated in the deeper layer and averaged in the $x$ direction. Units are in meters cubed per second squared. Dashed curves correspond to a wind stress perpendicular to the jet and solid curves to a wind stress parallel to the jet.

energy when the wind stress is parallel to the jet (Fig. 8 )! A nonnegligible part of the downward energy injection occurs outside the jet.

\section{c. Discussion}

The preceding results clearly display that the inertial resonance mechanism is still quite efficient to extract energy from the wind stress despite the presence of the dispersion mechanisms. One consequence of the horizontal propagation is that the energetic waves can travel against the wind far outside the jet while keeping extracting energy from it. Thus, the inertial resonance mechanism works over a much larger area than the jet area. Consequence of the downward propagation is that a nonnegligible part of the energy extracted from the wind is injected into the ocean interior.

A few simulations with the wind stress perpendicular to the jet (labeled TX2 through TX7 in Table 1 ) have been performed and compared with the preceding simulation (TX1) to get some qualitative insight on the sensitivity of the energy extracted from the wind to some parameters like the wind-stress magnitude and sign, the jet velocity and the Laplacian friction coefficient.

Results display a strong sensitivity on the wind-stress magnitude: total kinetic energy (in the surface and the deeper layers) after 20 inertial periods is almost 15 times larger for a wind-stress magnitude only 1.7 times larger (simulation TX6 in Table 1)! This agrees with the perturbation analysis that shows the growth rate dependence on the wind-stress magnitude. When the wind-stress sign is reversed, the total kinetic energy is almost unchanged (simulation TX2), although some qualitative changes occur. According to the criterion $k \tau_{x}<0$, exponentially growing inertial waves are now generated on the edges of the jet (where $\partial \zeta / \partial x<0$ ) and then propagate eastward. Consequence is that a strong kinetic energy increase is now observed in two distinct areas: in the negative vorticity region (as before) and also outside the jet but on the right side (Fig. 9a). The spatial distribution of the kinetic energy injected downward again appears to be a mirror of the kinetic energy increase in the upper layer (Fig. 9b). When the jet velocity magnitude $\left(V_{0}\right)$ is doubled, surprisingly, results do not display any changes at least for the mixed-layer kinetic energy (simulation TX5). An explanation is that, at the jet center, the exponential growth and the horizontal propagation balance for the same $k_{c}$ as the one found before, since its expression

$$
\approx \frac{6}{8} \frac{\tau}{f h^{2}} / \frac{g \Delta \rho}{\rho}
$$

does not depend on $V_{0}$. So energetic waves that mainly grow outside the jet should have the same wavenumber as before. The only difference is that the $k_{c}$ value is attained within a shorter time ( 2.5 inertial periods instead of 5) since $\partial \zeta / \partial x$ is larger. This leads to a slightly larger time duration for the energy growth outside the jet and consequently to an increase of the kinetic energy injected into the deeper layer. Increasing the density jump $\Delta \rho$ as well as the Laplacian friction coefficient $\nu$ leads to a smaller value of the total kinetic energy ex-

TABLE 1. Surface kinetic energy $\left(\mathrm{KE}_{s}\right)$ and bottom kinetic energy $\left(\mathrm{KE}_{b}\right)$ integrated over the whole domain at 20 inertial periods. Initial mixed-layer depth $\left(h_{i}\right)$ is $30 \mathrm{~m}$ for all simulations except for $T X 7$ where $h_{i}=26 \mathrm{~m}$.

\begin{tabular}{|c|c|c|c|c|c|c|c|c|c|c|}
\hline \multirow{3}{*}{$\frac{\text { Simulation }}{T X 1}$} & \multicolumn{5}{|c|}{ Parameters } & \multicolumn{5}{|c|}{ Results } \\
\hline & \multirow{2}{*}{$\frac{\tau_{x}\left(\times 10^{4}\right)}{1.2}$} & \multirow{2}{*}{$\frac{V_{0}}{0.15}$} & \multirow{2}{*}{$\frac{\nu}{40}$} & \multicolumn{2}{|c|}{$g \Delta \rho / \rho$} & \multicolumn{2}{|c|}{$\mathrm{KE}_{s}$} & \multicolumn{2}{|c|}{$\mathrm{KE}_{b}$} & \multirow{2}{*}{$\frac{\mathrm{KE}_{b} /\left(\mathrm{KE}_{s}+\mathrm{KE}_{b}\right)}{31.6 \%}$} \\
\hline & & & & 1.5 & $10^{-3}$ & 6.7 & $10^{-2}$ & 3.1 & $10^{-2}$ & \\
\hline$T X 2$ & -1.2 & 0.15 & 40 & 1.5 & $10^{-3}$ & 7.5 & $10^{-2}$ & 2.8 & $10^{-2}$ & $27.2 \%$ \\
\hline$T X 3$ & 1.2 & 0.15 & 40 & 3.0 & $10^{-3}$ & 5.5 & $10^{-2}$ & 1.3 & $10^{-2}$ & $19.1 \%$ \\
\hline$T X 4$ & 1.2 & 0.15 & 80 & 1.5 & $10^{-3}$ & 4.2 & $10^{-2}$ & 2.3 & $10^{-2}$ & $35.4 \%$ \\
\hline$T X 5$ & 1.2 & 0.30 & 40 & 1.5 & $10^{-3}$ & 6.8 & $10^{-2}$ & 6.4 & $10^{-2}$ & $48.5 \%$ \\
\hline$T \times 6$ & 2.0 & 0.15 & 40 & 1.5 & $10^{-3}$ & 6.0 & $10^{-1}$ & 8.0 & $10^{-1}$ & $57.1 \%$ \\
\hline$T X 7$ & 2.0 & 0.30 & 80 & 3.0 & $10^{-3}$ & 3.1 & $10^{-1}$ & 6.3 & $10^{-1}$ & $67.0 \%$ \\
\hline
\end{tabular}



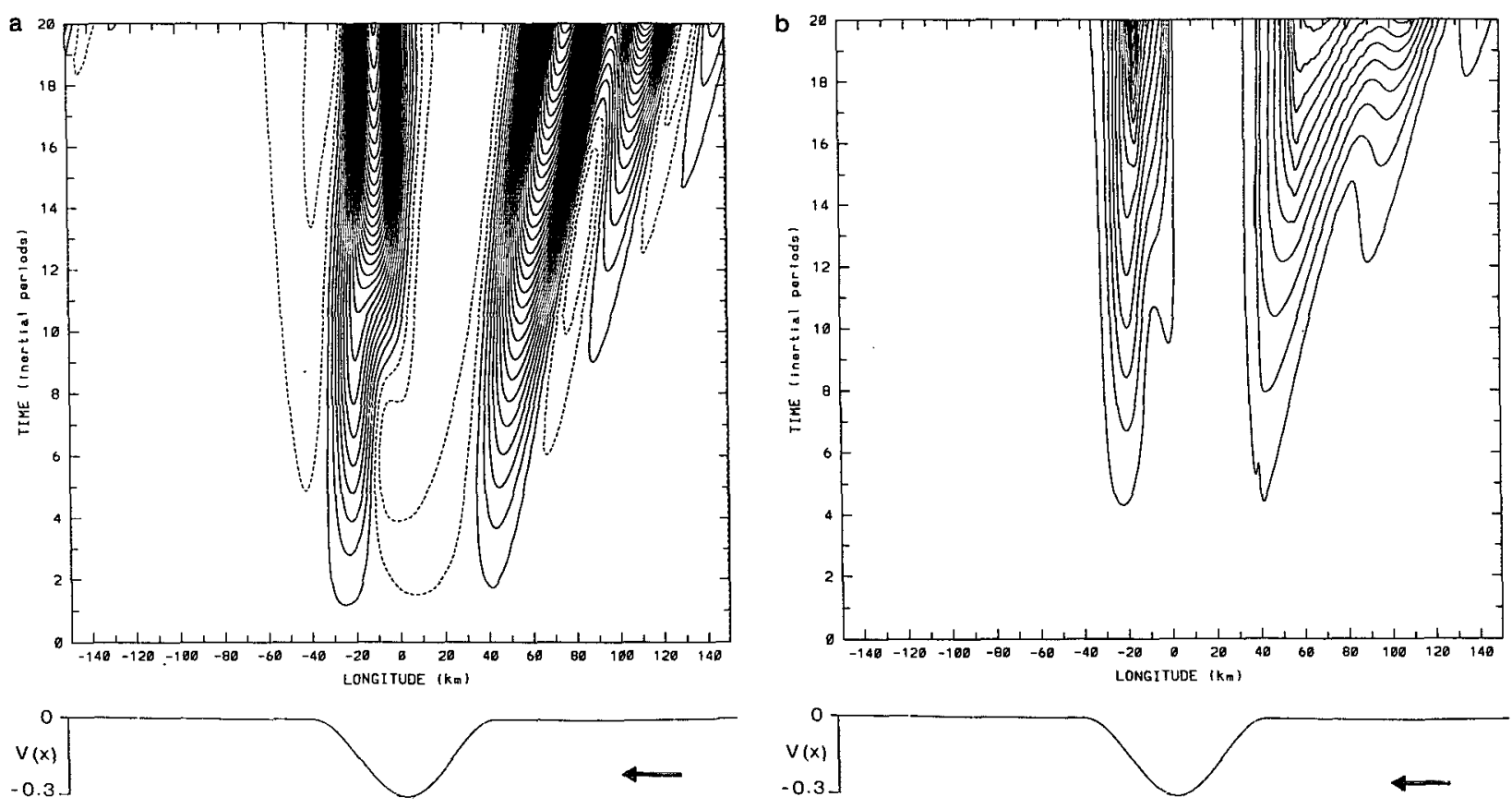

FIG. 9. Same as Fig. 7 but with a reversed wind-stress sign. Contours intervals are $0.01 \mathrm{~m}^{3} \mathrm{~s}^{-2}$ in both (a) and (b).

tracted from the wind (simulations TX3 and TX4). This energy decrease is simply due to the limitation of the exponential growth by the more significant dispersion mechanisms ( see appendix A).

These numerical results, using a 11/2-layer model, have provided useful information. A fully stratified model, however, is necessary to investigate the influence of thermocline structure on this resonance mechanism. Some numerical experiments performed with a primitive equation model are described in the next section.

\section{Numerical experiments with a $2 \mathrm{D}$ primitive equation model}

\section{a. The model}

The numerical model used is based on the SPEM code, described in Haidvogel et al. (1991). The code has been transformed to fit a two-dimensional geometry instead of a three-dimensional domain. The model uses the primitive equations with a linear equation of state depending on temperature only. The rigid-lid approximation is made. Finite differences are used in the horizontal and also in the vertical (instead of the spectral method). The turbulent fluxes in the mixed-layer processes are represented by a diffusion coefficient for momentum and temperature, which depends on the Richardson number following the classical level 2 model of Mellor and Yamada (1982). This parameterization makes use of the same physics as the Price et al. (1986) model. There are 114 levels in the vertical, with a grid spacing of $2 \mathrm{~m}$ down to $50 \mathrm{~m}$, gradually increasing to reach $50 \mathrm{~m}$ at $1000 \mathrm{~m}$, and then constant down to the bottom at $2000 \mathrm{~m}$. The high resolution in the top layers is necessary to ensure convergence of the mixed-layer parameterization scheme. It allows us to resolve such details as the Ekman spiral. The horizontal domain width is $600 \mathrm{~km}$ with a grid spacing of $1.5 \mathrm{~km}$. Experiments at $1-\mathrm{km}$ resolution have confirmed that the solutions have converged. The time step $(315 \mathrm{~s})$ is dictated by the vertical phase speed of large-scale internal waves.

In the regions of the model where the Richardson number is larger than the critical value, dissipation is kept as low as possible: there is a background vertical diffusion of $5.0 \times 10^{-5} \mathrm{~m}^{2} \mathrm{~s}^{-1}$. Horizontal diffusion is set to zero, but a biharmonic horizontal friction is used with coefficient $1.0 \times 10^{8} \mathrm{~m}^{4} \mathrm{~s}^{-1}$ for momentum and $5.0 \times 10^{7} \mathrm{~m}^{4} \mathrm{~s}^{-1}$ for temperature. So as to ensure that the barotropic streamfunction is periodic in the $x$ direction two sinusoidal and meridional jets, going in opposite directions and similar to the one used in section 4 , are studied at the same time in a domain 600 $\mathrm{km}$ wide. The wavelength is $80 \mathrm{~km}$, and the jet maximum velocity $0.3 \mathrm{~m} \mathrm{~s}^{-1}$. These barotropic jets are geostrophically balanced currents specified as initial conditions. Diffusion alone is too weak to affect these currents over a period of 15 days (i.e., 20 inertial periods). Note that no artificial decomposition into the Ekman layer and the geostrophic current is made; that is, interactions between inertial motions and these initially geostrophically balanced currents are fully taken into account. 
Different initial stratifications have been considered: in particular, one with a thin seasonal thermocline and one with a thick seasonal thermocline. The two-temperature profiles are plotted in Fig. 10. From both profiles the initial mixed-layer depth is $26 \mathrm{~m}$. The lower base of the thin seasonal thermocline is located at $40 \mathrm{~m}$.

\section{b. The results}

The model starts from rest, except for the barotropic jets, and is forced by a steady surface stress. The initial stratification considered corresponds to the temperature profile of Fig. 10 with a thin seasonal thermocline. Since the initial state is entirely barotropic, any upwelling or downwelling present in the solution is generated by the combined effect of the wind stress and the existing vorticity gradient. Again, two academic wind-stress situations are considered: one with a wind stress parallel to the jets $\left(\tau_{x}=0\right.$ and $\tau_{y}=2.0 \times 10^{-4}$ $\mathrm{m}^{2} \mathrm{~s}^{-2}$ ) and one with a wind stress perpendicular to the jets $\left(\tau_{x}=2.0 \times 10^{-4} \mathrm{~m}^{2} \mathrm{~s}^{-2}\right.$ and $\left.\tau_{y}=0\right)$. Only the results concerning the northward jet are examined in this section. Actually, the differences between the results concerning the southward jet and the ones related to the northward jet are in agreement with the ones found with the 11/2-layer model. The "northward case," however, better displays some interesting features of the vertical propagation of the inertial waves in the negative vorticity region.

\section{1) WIND STRESS PARALLEL TO THE JET}

Figure 11 shows the time-longitude plot of the $18^{\circ} \mathrm{C}$ isotherm depth (averaged over successive inertial periods to filter out the oscillations and enhance readability). The main signal is an upwelling at the jet center and downwelling on the edges. These upwellings

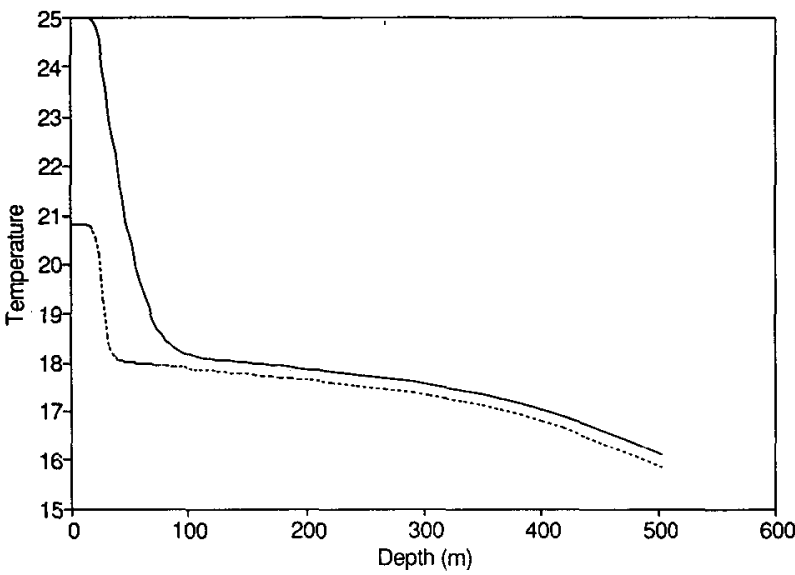

FIG. 10. Initial vertical temperature profiles, involving a thin (dashed line) and a thick (solid line) thermocline, used in simulations performed with the primitive equation model.

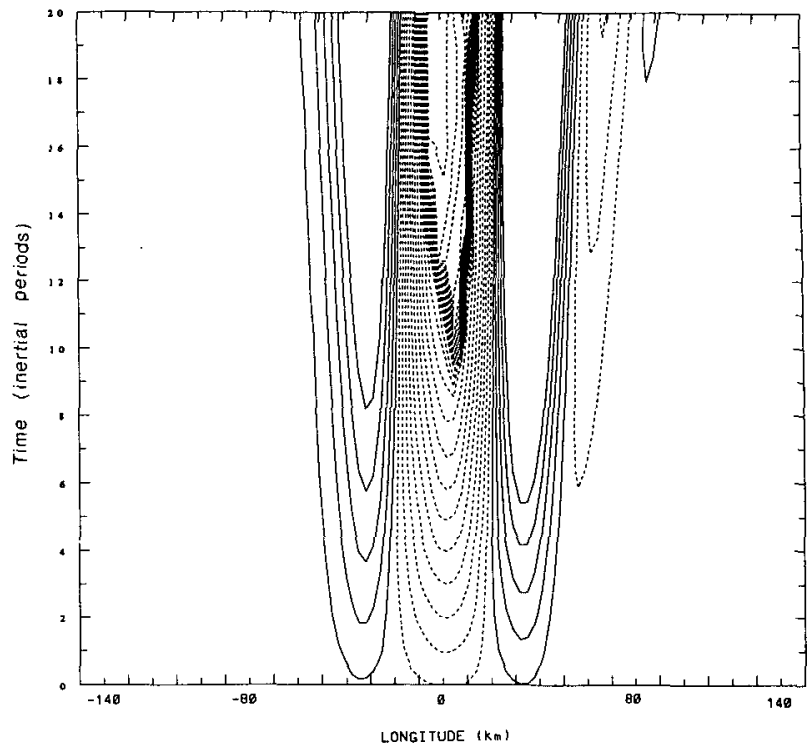

FiG. 11. Time-longitude plot of the $18^{\circ} \mathrm{C}$ isotherm depth when the wind stress is parallel to the jet. Mean depth is $57.5 \mathrm{~m}$. Dashed and continuous contours correspond respectively to negative and positive values. Contours interval is $1 \mathrm{~m}$.

and downwellings due to the divergence of the mean Ekman drift produce a total variation, after 20 inertial periods, of about $39 \mathrm{~m}$ of the isotherm initially located at $57.5 \mathrm{~m}$, that is, well below the mixed layer.

Figure 12 shows an isosurface of $u^{2}$ (plotted in a $x$ $z-t$ frame) whose value is $0.0002 \mathrm{~m}^{2} \mathrm{~s}^{-2}$. The energy is larger above this surface and smaller below. Each kinetic energy value on the $t$ axis is averaged over an inertial period. Outside the jet, the mean depth of the surface is quite close to the mixed-layer depth $(26 \mathrm{~m})$. In these regions, kinetic energy rapidly decreases below. On the edges of the jet, the kinetic energy surface attains $46 \mathrm{~m}$ after 20 inertial periods. So the inertial kinetic energy does not penetrate much in the deeper layers. On the other hand, the vertical velocity effects seem to extend to a much larger depth (at least up to $300 \mathrm{~m}$ in this simulation).

The kinetic energy averaged over the whole horizontal domain has been calculated, divided in two parts: the "surface" energy integrated from 0 to $40 \mathrm{~m}$, and the "bottom" energy integrated between $40 \mathrm{~m}$ and $2000 \mathrm{~m}$. More precisely, we use $u^{2}$ instead of $\left(u^{2}\right.$ $\left.+v^{2}\right) / 2$ as an estimate of the kinetic energy. This is because in the primitive equation model $v^{2}$ contains both an inertial and a geostrophic part that render it more difficult to interpret. In the surface layers (Fig. 13), kinetic energy decreases from $30.0 \times 10^{-2} \mathrm{~m}^{3} \mathrm{~s}^{-2}$ to $24.0 \times 10^{-2} \mathrm{~m}^{3} \mathrm{~s}^{-2}$. Note that this value, in this wind-stress situation, contains the energy related to the mean Ekman drift. Downward propagation of inertial energy is small: bottom energy attains $\approx 1.12 \times 10^{-2}$ $\mathrm{m}^{3} \mathrm{~s}^{-2}$ after 20 inertial periods. 


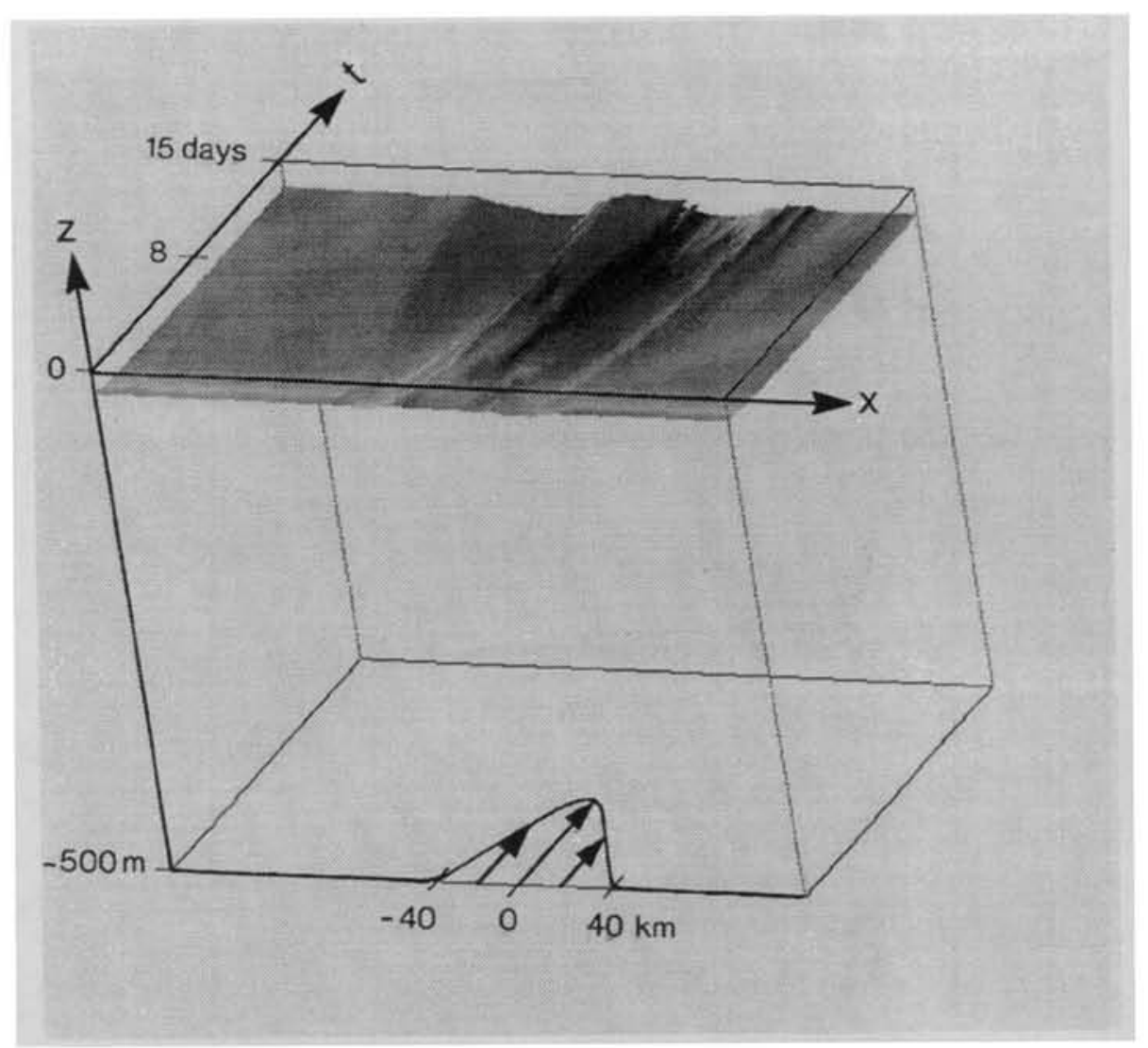

Fig. 12. Three-dimensional kinetic energy surface $\left(u^{2}\right)$ when the wind stress is parallel to the northward barotropic jet. Corresponding kinetic energy value is $0.0002 \mathrm{~m}^{2} \mathrm{~s}^{-2}$.

\section{2) WIND STRESS PERPENDICULAR TO THE JET}

With the same parameter settings as in the preceding simulation but a different wind-stress direction, the ocean response is completely different. First, the time-

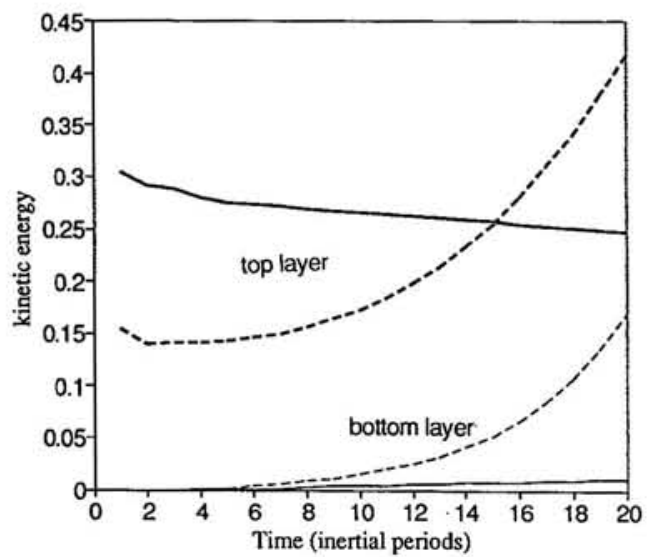

FIG. 13. Time series of kinetic energy $\left(u^{2}\right)$ integrated in the top layer $(0-40 \mathrm{~m})$ (thick lines) and in the bottom layer $(40-2000 \mathrm{~m})$ (thin lines) and averaged in the $x$ direction (units: $\mathrm{m}^{3} \mathrm{~s}^{-2}$ ). Dashed curves correspond to a wind stress perpendicular to the jet and solid curves to a wind stress parallel to the jet. longitude plot of the $18^{\circ} \mathrm{C}$ isotherm depth (not shown) does not display any significant variation after 20 inertial periods. But the most significant and conspicuous difference concerns the kinetic energy distribution within the ocean as well as its amount.

The $u^{2}$ isosurface (Fig. 14) is dramatically different from the one shown in Fig. 12, although the value is the same $\left(0.0002 \mathrm{~m}^{2} \mathrm{~s}^{-2}\right)$. It reveals a widespread penetration of energy at large depth. The contrast between the two experiments, which differ only by the wind direction, demonstrates the efficiency of the inertial resonance mechanism in this more realistic model. Outside the jet, on the right side, depth of the $u^{2}$ surface is still $26 \mathrm{~m}$, and in this region inertial energy drops to very small values just below this depth. Inside the jet (mainly on the edges) and outside the jet (on the left side) this surface penetrates downward to reach a depth greater than $500 \mathrm{~m}$. One quite interesting feature displayed by Fig. 14 is the conspicuous concentration of kinetic energy in the deeper layers, which appears in the negative vorticity region and that seems to be trapped there. This feature is not so well displayed in the "southward jet case," since the negative vorticity region is next to the upwind region, outside the jet, where growing inertial waves propagate. Since the inertial resonance mechanism can work out of the jet in 


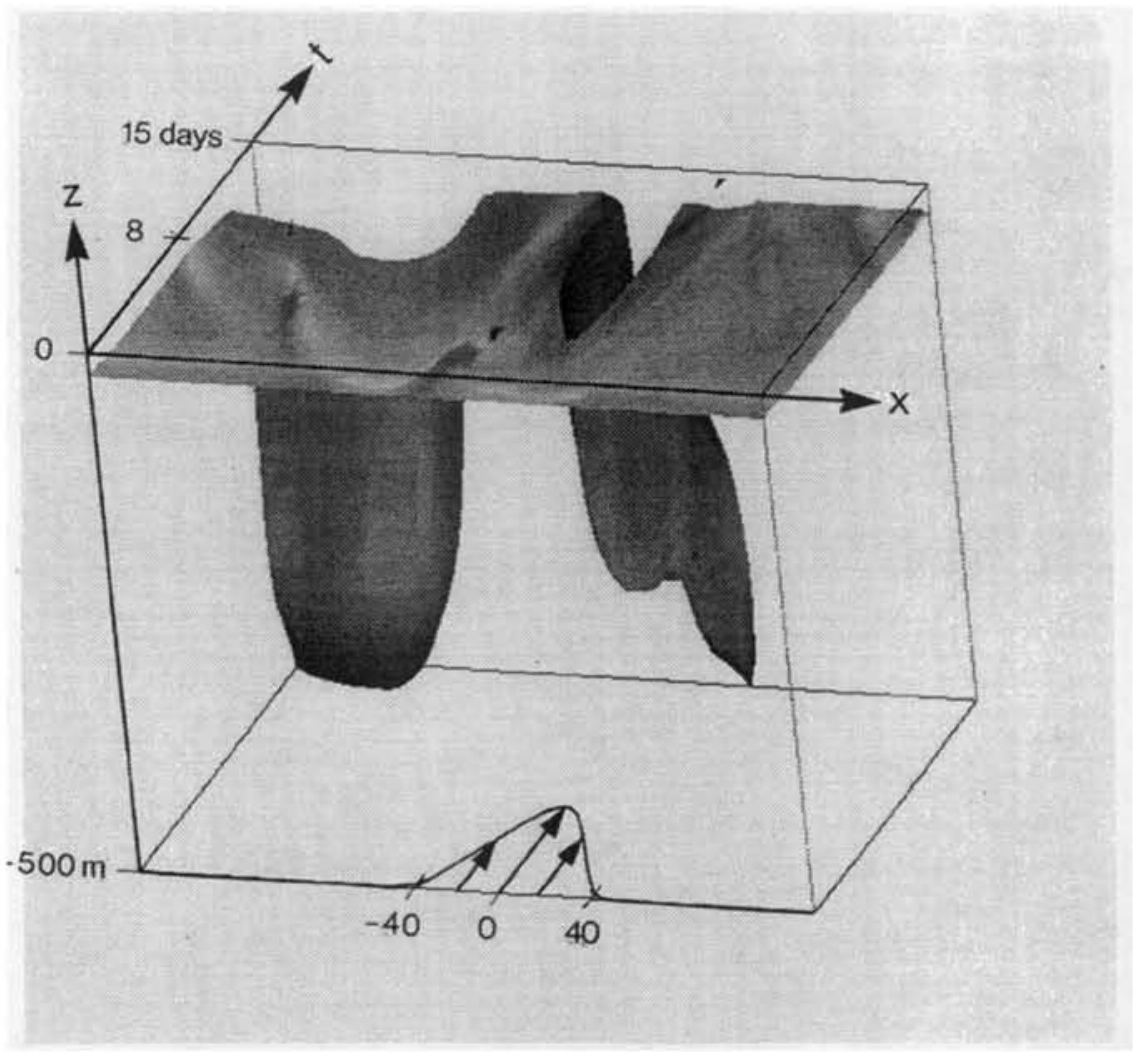

FIG. 14. Three-dimensional kinetic energy surface $\left(u^{2}\right)$ when the wind stress is perpendicular to the northward barotropic jet. Corresponding kinetic energy value is $0.0002 \mathrm{~m}^{2} \mathrm{~s}^{-2}$.

the surface layers, the large amount of kinetic energy extracted from the wind is spread on a much larger area than the jet area. Figure 15 confirms the inertial motions are growing in the surface layers outside the jet (Fig. 15a), and that a large amount of the kinetic energy is dispersed into the deeper layers (Fig. 15b). Note that these features qualitatively agree with the results found with the simplified model in section 4 when the wind-stress orientation relatively to the jet was the same (see Fig. 9).

Time evolution of the total kinetic energy (estimated from $u^{2}$ ) is shown in Fig. 13 to allow comparison with the previous case. The difference with the preceding case $\tau_{y} \neq 0$ is quite significant. The total kinetic energy extracted from the wind (i.e., the one in the surface layers plus the one in the deeper layers) attains 56.0 $\times 10^{-2} \mathrm{~m}^{3} \mathrm{~s}^{-2}$ after 20 inertial periods. This is almost 3.7 times larger than if there was no jet. Kinetic energy evolution in the surface layers displays an almost linear increase starting after 3 days. After 15 days ( 20 inertial periods) the surface energy is 2.5 times larger than in the first inertial periods. Kinetic energy evolution in the deeper layers has more an exponential-like behavior. The value attained after 20 inertial periods is 17.0 $\times 10^{-2}$, that is, 15 times larger than the corresponding one when the wind stress is parallel to the jet.

\section{c. Discussion}

Some other numerical simulations, using the primitive equation model, have been performed in order to assess the efficiency of the inertial resonance mechanism to different physical characteristics, such as the vertical structure of the geostrophic jet and the vertical structure of the seasonal thermocline.

First, a simulation was performed with baroclinic jets similar to the barotropic ones in the upper layers but decaying below $200 \mathrm{~m}$ (Fig. 16). Using the same mixed-layer depth and seasonal thermocline as before as well as the same wind stress, the results do not display any changes for the kinetic energy distribution in the upper layers (Fig. 17a) as well as in the deeper layers (Fig. 17b). The only change concerns the vertical propagation of the inertial waves in the negative vorticity region, which attains a depth of $200 \mathrm{~m}$ instead of $500 \mathrm{~m}$. Time evolution of the total kinetic energy compares well with the one corresponding to the barotropic jet: total kinetic energy after 15 days attains a value of $0.38 \mathrm{~m}^{3} \mathrm{~s}^{-2}$ (instead of $0.39 \mathrm{~m}^{3} \mathrm{~s}^{-2}$ ) in the upper layers and $0.23 \mathrm{~m}^{3} \mathrm{~s}^{-2}$ (instead of $0.17 \mathrm{~m}^{3} \mathrm{~s}^{-2}$ ) in the deeper layers. Therefore, the baroclinity of the jet does not affect much the efficiency of the inertial resonance mechanism. 

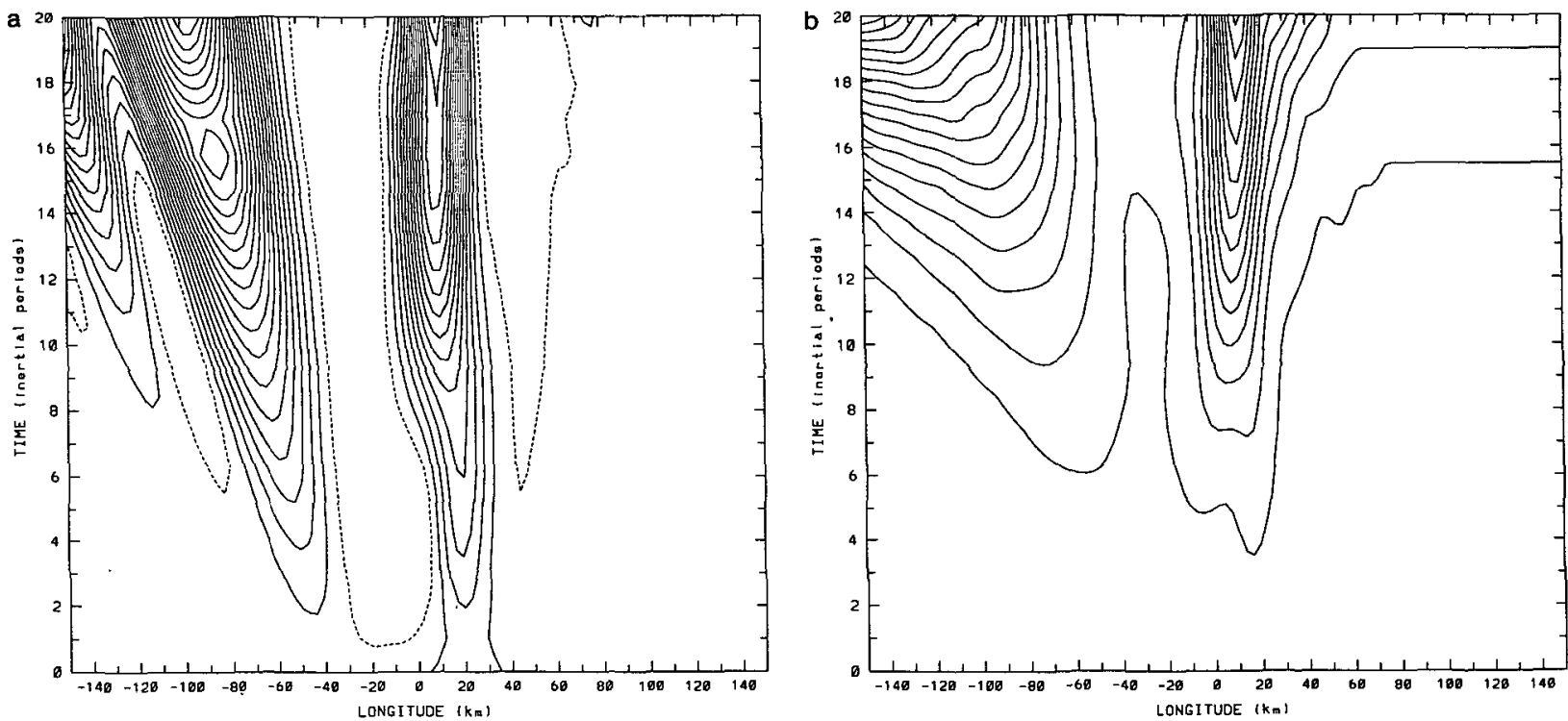

FIG. 15. Time-longitude plot of the kinetic energy $\left(u^{2}\right)$ relatively to a mean value, averaged over one inertial period and integrated (a) in the top layer $(0-40 \mathrm{~m})$ and $(\mathrm{b})$ in the bottom layer $(40-2000 \mathrm{~m})$ when the wind stress is perpendicular to the jet. Dashed and continuous contours correspond, respectively, to negative and positive values. Contours intervals are $0.04 \mathrm{~m}^{3} \mathrm{~s}^{-2}$ in (a) and $0.02 \mathrm{~m}^{3} \mathrm{~s}^{-2}$ in (b). Mean value [equal to $1.410^{-1} \mathrm{~m}^{3} \mathrm{~s}^{-2}$ in (a) and zero in (b)] corresponds to the value when no jet is present.

An experiment using again the barotropic jets has been run with a thicker seasonal thermocline (see Fig. 10 ). In the latter case the amount of energy radiated downward is dramatically reduced (by a factor of 5), and the contrast between wind stress parallel and perpendicular to the front is no longer dramatic. This is easy to understand considering that the horizontal dispersion energy flux and the downward energy flux depend, respectively, on the square and on the cube of the vertical wavenumber. Indeed, a larger thermocline

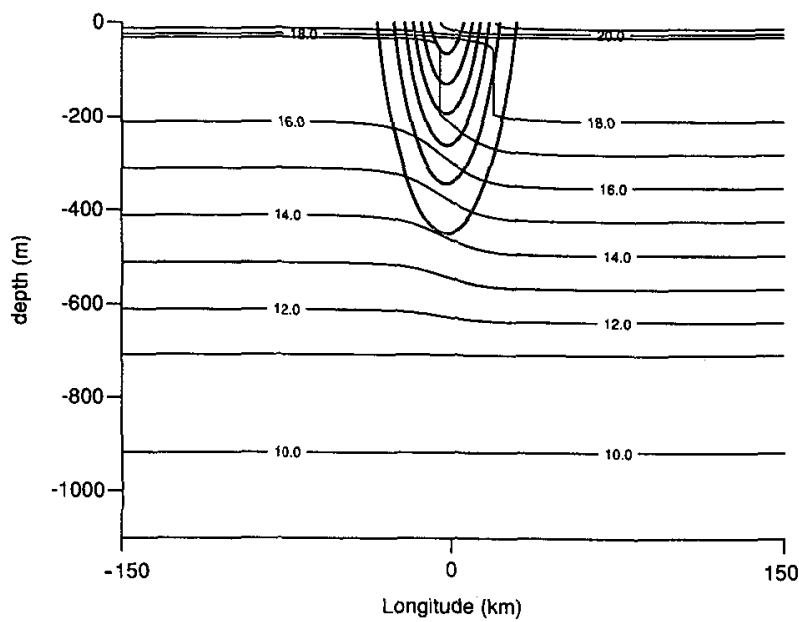

FIG. 16. Initial cross-frontal distribution of temperature (thin lines) and along-front velocity (thick lines) used for the northward baroclinic jet case. Temperature are in degrees Celsius. Velocity contours are $5 \mathrm{~cm} \mathrm{~s}^{-1}$ and maximum contour in the jet core is $30 \mathrm{~cm} \mathrm{~s}^{-1}$. thickness leads to a smaller vertical wavenumber and therefore to a stronger effect of the dispersion mechanisms. In particular, the increased vertical propagation becomes large enough to prevent the development of inertial resonance. This tendency was clearly revealed by the short sensitivity study performed in section $4 d$ (see also Table 1).

In the simple 1 1/2-layer model, the inertial resonance completely disappears when the forcing enters over a constant depth $\left(\tau / h_{i}\right.$ instead of $\left.\tau / h\right)$. This suggests that the mixed-layer parameterization, which lets the depth of penetration of the forcing vary in time and space, plays an essential role in the primitive equation model. This is true, but the issue is not as simple as with the one-layer model. An experiment has been run without any mixed-layer parameterization, and the wind entered as a body force over a depth of $26 \mathrm{~m}$. The amount of energy in the deeper layers was reduced by a factor of 2 , but the patterns of kinetic energy generation were unchanged. The inertial resonance phenomenon was still possible in that case because of the nonlinear terms $w \partial u / \partial z, w \partial v / \partial z$ in the momentum equations, which are absent in the $11 / 2$-layer model. On the other hand, when all nonlinear terms were removed from the momentum equations (except of course for the modification of the inertial frequency due to the geostrophic jet), the inertial resonance phenomenon disappeared completely. The energy in the bottom layers was reduced by a factor of 10 compared with the fully nonlinear case. Moreover, since the waves propagating outside the jet could no longer amplify, the downward energy injection occurred almost exclusively below the jet. 

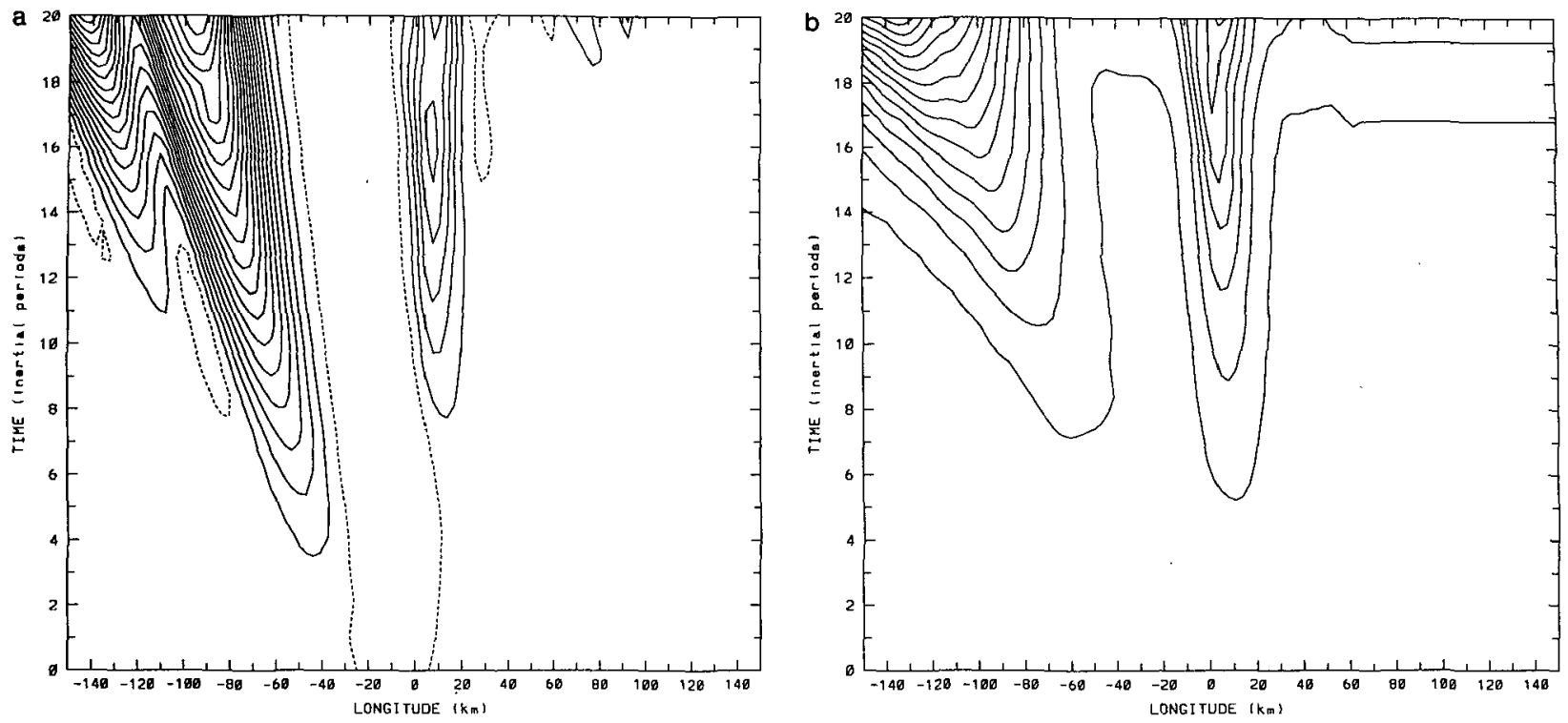

Fig. 17. Same as Fig. 15 but for a baroclinic jet. Contours intervals are $0.07 \mathrm{~m}^{3} \mathrm{~s}^{-2}$ in (a) and $0.05 \mathrm{~m}^{3} \mathrm{~s}^{-2}$ in (b). Mean value [equal to $1.4 \times 10^{-1} \mathrm{~m}^{3} \mathrm{~s}^{-2}$ in (a) and zero in (b)] corresponds to the value when no jet is present.

\section{Conclusions}

This study has examined how wind-driven inertial motions can be affected by the vorticity field of mesoscale oceanic structures. A geostrophic and barotropic jet has been chosen as the simplest example.

Linear analysis reveals that the jet vorticity affects both the amplitude and the phase of the inertial motions. Consequences of these features for the linear dynamics have been described in the literature. The effect on the phase, however, induces a time-increasing spatial variability, with smaller and smaller wavenumbers. Consequently, the nonlinear terms become important, even if they can be ignored initially. This was the main motivation that led us to investigate their effects.

A perturbation analysis of the nonlinear equations of a simple model has revealed an exponential growth of the wind-driven inertial motions. The main part of this exponential growth is due to an inertial resonance mechanism and is related to the wind-stress component perpendicular to the jet. This resonance happens when the true depth $h(x, t)$ is taken into account in the forcing term $\tau_{x} / h$ present in the momentum equations. In these conditions the phase relationship of the velocity component $u$ and mixed-layer depth $h$ allows waves propagating against the wind to extract energy from it and to grow.

Results from a simple numerical model involving the full nonlinear equations have confirmed the existence of this inertial resonance mechanism. Competition between inertial resonance and the horizontal and vertical propagation of the inertial waves induced by the underneath stratification has also been assessed. One consequence of this competition is that energetic inertial waves horizontally propagate against the wind and keep growing well outside the geostrophic jet (insofar as the downward injection in the deeper layers is not too large). Therefore, the kinetic energy is spread over a much larger area. Another consequence is that a large part of this energy is injected into the ocean interior. All these results have been confirmed using a 2D primitive equation model with a realistic stratification involving a shallow mixed layer and a thin seasonal thermocline. This more complete model has shown that the penetration of the inertial energy within the ocean interior is much enhanced when inertial resonance is active.

This approach, using a perturbation analysis of the nonlinear equations, a simple numerical model, and a more realistic primitive equation model, has led us to be confident about the existence of the inertial resonance mechanism. Although the mesoscale jet considered in this study is the simplest mesoscale structure, these results should be valid for other structures as mesoscale eddies. The only necessary "ingredient" is a nonzero vorticity field with large enough gradients to induce a spatial variability of the inertial motions. Then, if the wavenumber of the inertial motions has a nonzero component parallel to the wind and if the wind duration is equal or larger than a few inertial periods, the inertial resonance mechanism is able to extract energy from the wind. One consequence is that, because of the downward energy injection into the ocean interior, a large part of this energy is available for deep mixing.

The physics behind this inertial resonance mechanism suggests that it should work in other situations (Tréguier and Klein 1993), whenever the surface in- 
ertial oscillations vary in space with a nonzero wavenumber parallel to the steady wind stress. This should be the case, for example, near a coast with a nonzero onshore wind stress as seen in the numerical results of Arhan (1973).

Finally, while we are able to find the inertial resonance in many numerical solutions, nevertheless, we have not found any observations in the literature that would confirm (or refute) the existence of such mechanism. Our theoretical and numerical study shows that the efficiency of the inertial resonance results from the "net" growth rate, that is, the difference between the inertial resonance growth rate and the injection rate of energy in the deeper layers due to the vertical propagation of the inertial waves. The inertial resonance growth rate is related to the ratio of the wind-stress magnitude to the mixed-layer depth and to the horizontal wavenumber. The downward injection rate is linked to the horizontal wavenumber as well, but mainly to the vertical wavenumber related to the seasonal thermocline thickness. Hence, the inertial resonance mechanism is found to be quite efficient with a shallow mixed layer and a thin thermocline (that correspond to spring conditions), whereas its efficiency is dramatically reduced when a thick thermocline is considered. Another necessary "ingredient" is the wind time duration. With the parameters we used, the growth effects can be observed only if the mean wind persists for periods up to 10 or 15 days. Finally, our results have revealed that the main significant effects of the inertial resonance mechanism occur not within the geostrophic jet area, but well outside the jet. These comments suggest that the inertial resonance mechanism could be observed only in some particular situations.

Acknowledgments. This work is supported by IFREMER and CNRS. Some of the calculations reported here were done at the Centre de Calcul Vectoriel pour la Recherche (Palaiseau, France). We thank Bach Lien Hua and Kevin Speer for useful comments on an earlier version of this paper. We are particularly grateful to Jim Price for many insightful discussions and comments on the manuscript.

\section{APPENDIX A}

\section{Asymptotic Solution within the Geostrophic Jet}

Let us consider Eq. (5) with $D_{h u}$ and $D_{h v}$ parameterized as a Laplacian friction with a friction coefficient $\nu$, acting only on the oscillating components of the Ekman transport. We use the same scalings as in section $2 \mathrm{~b}$, with in addition:

$$
g^{\prime}=g \frac{\Delta \rho}{\rho} \frac{h_{i}}{f U L}, \quad \nu^{\prime}=\frac{\nu}{U L},
$$

and we assume that $g^{\prime} \approx \nu^{\prime}$ are of order $O(1)$. Then using the same formalism as the one used in section
$3 \mathrm{a}$, we get the following nondimensional linearized equations for the oscillating components of the Ekman transport:

$$
\begin{gathered}
\frac{\partial u}{\partial t}-v+2 \epsilon \frac{\tau_{y}}{\omega^{2}} \frac{\partial u}{\partial x}+2 \epsilon u \frac{\partial \tau_{y} / \omega^{2}}{\partial x} \\
-\epsilon \nu^{\prime} \frac{\partial^{2} u}{\partial x^{2}}+\epsilon g^{\prime} \frac{\partial h_{1}}{\partial x}=0 \\
\frac{\partial v}{\partial t}+\omega^{2} u+\epsilon \frac{\tau_{y}}{\omega^{2}} \frac{\partial v}{\partial x}-\epsilon \tau_{x} \frac{\partial u}{\partial x} \\
+\epsilon v \frac{\partial \tau_{y} / \omega^{2}}{\partial x}-\epsilon \nu^{\prime} \frac{\partial^{2} v}{\partial x^{2}}=0 \\
\frac{\partial h_{1}}{\partial t}=-\frac{\partial u}{\partial x}
\end{gathered}
$$

They can be rewritten as

$$
\begin{aligned}
& \frac{\partial^{2} u}{\partial t^{2}}+\omega^{2} u+3 \epsilon \frac{\tau_{y}}{\omega^{2}} \frac{\partial^{2} u}{\partial t \partial x}+3 \epsilon \frac{\partial \tau_{y} / \omega^{2}}{\partial x} \frac{\partial u}{\partial t} \\
&-\epsilon \tau_{x} \frac{\partial u}{\partial x}-2 \epsilon \nu^{\prime} \frac{\partial^{3} u}{\partial t \partial x^{2}}-\epsilon g^{\prime} \frac{\partial^{2} u}{\partial x^{2}}=0
\end{aligned}
$$

Let us look for solutions like

$$
u \approx u_{0}(x) e^{-i\left(\omega t+f_{1}\right)}
$$

with $f_{1}=f_{1}(x, t)$ a complex function (representing a phase perturbation ) to be determined.

Using (24) into (23), when only terms of order $O(1)$ and $O(\epsilon)$ are retained we get (with $f_{2}=\partial f_{1} / \partial t$ )

$$
\begin{aligned}
& 2 \omega f_{2}+i \frac{\partial f_{2}}{\partial t}=-3 \frac{\tau_{y}}{\omega} \frac{\partial \omega}{\partial x} t+g^{\prime}\left(\frac{\partial \omega}{\partial x}\right)^{2} t^{2} \\
& -i\left[3 \omega \frac{\partial \tau_{y} / \omega^{2}}{\partial x}-\tau_{x} \frac{\partial \omega}{\partial x} t+2 \nu^{\prime} \omega\left(\frac{\partial \omega}{\partial x}\right)^{2} t^{2}\right]
\end{aligned}
$$

The asymptotic solution (i.e., for large $t$ ) of (25) is

$$
\begin{aligned}
f_{2} \approx & -\frac{3}{2} \frac{\tau_{y}}{\omega^{2}} \frac{\partial \omega}{\partial x} t+\frac{g^{\prime}}{2 \omega}\left(\frac{\partial \omega}{\partial x}\right)^{2} t^{2}+i\left[\frac{\tau_{x}}{2 \omega} \frac{\partial \omega}{\partial x} t\right. \\
& \left.-\frac{3}{2} \frac{\partial \tau_{y} / \omega^{2}}{\partial x}-\frac{g^{\prime}}{2 \omega^{2}}\left(\frac{\partial \omega}{\partial x}\right)^{2} t-\nu^{\prime}\left(\frac{\partial \omega}{\partial x}\right)^{2} t^{2}\right],
\end{aligned}
$$

which leads to

$$
\begin{aligned}
f_{1} \approx & -\frac{3}{4} \frac{\tau_{y}}{\omega^{2}} \frac{\partial \omega}{\partial x} t^{2}+\frac{g^{\prime}}{6 \omega}\left(\frac{\partial \omega}{\partial x}\right)^{2} t^{3}+i\left[\frac{\tau_{x}}{4 \omega} \frac{\partial \omega}{\partial x} t^{2}\right. \\
& \left.-\frac{3}{2} \frac{\partial \tau_{y} / \omega^{2}}{\partial x} t-\frac{g^{\prime}}{4 \omega^{2}}\left(\frac{\partial \omega}{\partial x}\right)^{2} t^{2}-\frac{\nu^{\prime}}{3}\left(\frac{\partial \omega}{\partial x}\right)^{2} t^{3}\right]
\end{aligned}
$$

Note that the $g^{\prime}$ and $\nu^{\prime}$ terms are both damping terms. Moreover, values used are such that $g^{\prime} \approx \nu^{\prime}$. Consequently, the $\nu^{\prime}$ terms dominate the $g^{\prime}$ terms in Eq. (27) since they evolve as $t^{3}$. As a result, we have 


$$
\begin{aligned}
f_{1} \approx & -\frac{3}{4} \frac{\tau_{y}}{\omega^{2}} \frac{\partial \omega}{\partial x} t^{2}+\frac{g^{\prime}}{6 \omega}\left(\frac{\partial \omega}{\partial x}\right)^{2} t^{3} \\
& +i\left[\frac{\tau_{x}}{4 \omega} \frac{\partial \omega}{\partial x} t^{2}-\frac{3}{2} \frac{\partial \tau_{y} / \omega^{2}}{\partial x} t-\frac{\nu^{\prime}}{3}\left(\frac{\partial \omega}{\partial x}\right)^{2} t^{3}\right],
\end{aligned}
$$

which in dimensional form becomes

$$
\begin{aligned}
& \epsilon f_{1} \approx-\frac{3}{8} \frac{\tau_{y}}{h_{i}(f+\zeta)} \frac{\partial \zeta}{\partial x} t^{2}+\frac{g}{24} \frac{\Delta \rho}{\rho} \frac{h_{i}}{f}\left(\frac{\partial \zeta}{\partial x}\right)^{2} t^{3} \\
& +i\left[\frac{\tau_{x}}{8(f+\zeta) h_{i}} \frac{\partial \zeta}{\partial x} t^{2}-\frac{3}{2 h_{i}} \frac{\partial \tau_{y} /(f+\zeta)}{\partial x} t\right. \\
& \left.-\frac{\nu}{12}\left(\frac{\partial \zeta}{\partial x}\right)^{2} t^{3}\right]
\end{aligned}
$$

Note that, as explained in section 3a, the asymptotic expansion is valid only for the first inertial periods. For times larger than 10 inertial periods, we have strong experimental evidence that the solution is discontinuous within the jet for $h v, \partial h u / \partial t, \partial h u / \partial x$ in the limit $g^{\prime}, \nu^{\prime} \rightarrow 0$. This can be guessed, for example, from the cusplike behavior of $h u$ in Fig. 6 . We have not, however, been able to prove it analytically.

\section{APPENDIX B}

\section{Parameterization of the Dispersion Mechanisms and of the Turbulent Entrainment}

Parameterizations of the vertical propagation are suggested by the energy equation. The energy flux at the base of the mixed layer can be estimated as $\mathscr{F}$ $=-C_{g z} K_{e}$, with $C_{g z}$ the vertical group velocity and $K_{e}$ $=\left(u^{2}+v^{2}\right) / 2$. For near-inertial waves $C_{g z}$ $\approx-\left(N^{2} / f\right) k_{x}^{2} / k_{z}^{3}$, with $N^{2}$ the Brunt-Väisälä frequency, $k_{x}$ the horizontal wavenumber, and $k_{z}$ the vertical wavenumber. In the ocean, vertical dispersion below the mixed layer depends on the stratification in the seasonal thermocline. The largest dispersion occurs for a strong thermocline ( $N^{2}$ large) and for the smallest $k_{z}$, that is, a large vertical scale corresponding to a large thermocline thickness. If $k_{z}$ is imposed by the stratification, then the energy flux is best parameterized by a Laplacian friction (see Gill 1984) with coefficient $\nu$ (since the flux due to Laplacian friction is also proportional to $k_{x}^{2}$ ):

$$
\mathcal{F}=2 \nu k_{x}^{2} h K_{e}=\frac{N^{2}}{f} \frac{k_{x}^{2}}{k_{z}^{3}} K_{e} .
$$

The expression for $\nu$ deduced from this equality strongly depends on $k_{z}$. For typical values of $N^{2}, f$ and $h$, one can find values of $\nu$ ranging from about $50 \mathrm{~m}^{2} \mathrm{~s}^{-1}$ (for $k_{z}^{-1}=20 \mathrm{~m}$ ), up to $400 \mathrm{~m}^{2} \mathrm{~s}^{-1}$ (for $k_{z}^{-1}=40 \mathrm{~m}$ ). The critical influence of the seasonal thermocline thickness in the vertical dispersion of inertial energy is confirmed by our experiments using a primitive equation model (section 5c). Therefore, the Laplacian fric- tion has been used. The kinetic energy dissipated by the Laplacian friction (which is assumed to be transferred into the deeper layer) when integrated over the whole domain $(\Omega)$ is calculated by

$$
K_{e d}(t)=-\nu \int_{0}^{t} \int_{\Omega} h\left[\left(\frac{\partial u}{\partial x}\right)^{2}+\left(\frac{\partial v}{\partial x}\right)^{2}\right] d x d t .
$$

Another possible parameterization of vertical dispersion would be a linear friction with coefficient $r$. For an inertial wave with fixed frequency $\omega$, the phase angle $\alpha=k_{x} / k_{z}$ is constant. Therefore, the group velocity is $C_{g z}=-\left(N^{2} / \omega\right) \alpha^{2} / k_{z}$ and the energy flux

$$
\mathcal{F}=-\frac{N^{2}}{\omega} \frac{\alpha^{2}}{k_{z}} K_{e}=-r h K_{e}
$$

does not depend on the horizontal scale of the motion. The corresponding values for $r$ range from 1 to 10 days. A linear friction, however, cannot balance the $\exp \left(t^{2}\right)$ growth of the solution generated by inertial resonance. In numerical experiments with linear friction, the oscillations first decay until the $\exp \left(t^{2}\right)$ coefficient is larger than the decay factor $\exp (-r t)$. When $r$ is large, this happens for later times. We have not, however, found a threshold value of $r$ above which the growth would be suppressed (for the cases with no horizontal dispersion): hence our choice of Laplacian friction.

The turbulent entrainment at the base of the mixed layer has been considered. Parameterization used for the entrainment velocity $w_{e}$ involves (as in Klein and Hua 1988; see also Price et al. 1986) a critical mixedlayer depth $h_{c}$ such that

$$
w_{e}=\left\{\begin{array}{cl}
a\left(h-h_{c}\right), & h<h_{c} \\
0, & h \geqslant h_{c}
\end{array}\right.
$$

with an $e$-folding time $a^{-1}=f^{-1}$. This critical depth $h_{c}$, calculated at each time step from

$$
h_{c}=\left[\frac{\operatorname{Ri}_{c}\left[(h u)^{2}+(h v)^{2}\right]}{g \frac{\Delta \rho}{\rho}}\right]^{1 / 3},
$$

involves a critical Richardson number following Pollard et al. ( 1973) and Price et al.'s ( 1986) ideas. In the simplified model used $g \Delta \rho / \rho$ does not need to be the same for the entrainment parameterization and the horizontal propagation. Propagation depends mainly on the total density jump in the seasonal thermocline whereas entrainment depends on the local stratification at the base of the mixed layer. Instead of specifying both the critical Richardson number $\mathrm{Ri}_{c}$ and $g \Delta \rho / \rho$, we have calculated their ratio such the maximum value of $h_{c}$ is equal to $h_{c o}=20 \mathrm{~m}$ when no jet is present. Since $h_{i}=30 \mathrm{~m}$, no entrainment happens initially but it occurs only at the later stages when the mixed-layer depth reaches smaller values because of the Ekman pumping effects. 


\section{REFERENCES}

Arhan, M., 1973: Etude nonlineaire des ondes internes dans un milieu a deux couches fluides en rotation. Rapp. CNEXO, 17.

Gill, A. E., 1984: On the behavior of internal waves in the wakes of storms. J. Phys. Oceanogr., 14, $1129-1151$.

Haidvogel, D. B., J. L. Wilkin, and R. Young, 1991: A semi-spectral primitive equation ocean circulation model using vertical sigma and orthogonal curvilinear horizontal coordinates. J. Comput. Phys., 94, 151-185.

Klein, P., and B. L. Hua, 1988: Mesoscale variability of the winddriven mixed layer: Influence of a quasigeostrophic flow. J. Mar. Res., 46, 495-525.

_- and -1990 : The mesoscale variability of the sea surface temperature: An analytical and numerical model. J. Mar. Res., 48, 729-763.

Kunze, E., 1985: Near-inertial wave propagation in geostrophic shear. J. Phys. Oceanogr.. 15, 544-565.

in a front. J. Phys. Oceanogr., 14, 566-581.

Large, W. G., J. C. McWilliams, and P. P. Niiler, 1986: Upper ocean thermal response to strong autumnal forcing of the Northeast Pacific. J. Phys. Oceanogr., 16, 1524-1550.

Mellor, G. L., and T. Yamada, 1982: Development of turbulence closure model for geophysical fluid problems. Rev. Geophys. Space Phys., 20, 851-875.
Niiler, P. R., 1969: On the Ekman divergence in an oceanic jet. $J$ Geophys. Res., 74(28), 7048-7052.

Pollard, R. T., 1990: Large potential vorticity variations at small scales in the upper ocean. Nature.

Pollard, R. T., P. B. Rhines, and R. O. R. Y. Thompson, 1973: The deepening of the wind-mixed layer. Geophys. Fluid Dyn., 4, $381-404$.

Price, J. F., R. A. Weller, and R. Pinkel, 1986: Diurnal cycling: Observations and models of the upper ocean response to diurnal heating, cooling and wind mixing. J. Geophys. Res., 91(C7), 8411-8427.

Rubenstein, D. H., 1983: Vertical dispersion of inertial waves in the upper ocean. J. Geophys. Res., 88, 4368-4380.

- - and G. O. Roberts, 1986: Scattering of inertial waves by an ocean front. J. Phys. Oceanogr., 16, 121-131.

Tréguier, A. M., and P. Klein, 1993: Nonlinear amplification of the wind-driven inertial motions. J. Fluid Mech., submitted.

Weller, R. A., 1982: The relation of near-inertial motions observed in the mixed layer during the JASIN (1978) experiment to the local wind stress and to the quasi-geostrophic flow field. J. Phys. Oceanogr., 12, 1122-1136.

- 1985 : Near surface velocity variability at inertial and subinertial frequencies in the vicinity of the California current. $J$. Phys. Oceanogr., 15, 372-385. 